\title{
Bu-Shen-Zhu-Yun decoction induces PRLR deubiquitination and JAK2/STAT5 activation via CSN5 in vitro
}

\author{
Hua Feng ${ }^{1,2}$, Huifang Zhou ${ }^{3, \&}$, Jianxia Lu ${ }^{1}$, Qing Zhang ${ }^{4}$ Xingran Tang ${ }^{5}$, Yujie Shang ${ }^{5}$ \\ ${ }^{1}$ Institute of Rehabilitation, Jiangsu Vocational College of Medicine, Yancheng 224008, Jiangsu Province, China \\ ${ }^{2}$ Department of Gynecology, Nanjing University of Chinese Medicine, Nanjing 224005, Jiangsu Province, China \\ ${ }^{3}$ Department of Gynecology, Affiliated Hospital of Nanjing University of Chinese Medicine, Nanjing 210029, \\ Jiangsu Province, China \\ ${ }^{4}$ School of Clinical Medicine Jiangsu Vocational College of Medicine, Yancheng 224008, Jiangsu Province, China \\ ${ }^{5}$ Nanjing University of Chinese Medicine, Nanjing 224005, Jiangsu Province, China
}

Correspondence to: Huifang Zhou; email: zhouhuifang2011301@163.com, https://orcid.org/0000-0001-5208-8727

Keywords: Bu-Shen-Zhu-Yun decoction, hyperprolactinemia infertility

Received: December 2, 2020

Accepted: May 11, 2021

Published: August 23, 2021

Copyright: (C) 2021 Feng et al. This is an open access article distributed under the terms of the Creative Commons Attribution License (CC BY 3.0), which permits unrestricted use, distribution, and reproduction in any medium, provided the original author and source are credited.

\section{ABSTRACT}

Purpose: To determine the effect of Bu-Shen-Zhu-Yun Decoction (BSZY-D) on the kisspeptin through JAK2/STAT5 signaling pathway in hyperprolactinemia (HPRL) infertility.

Method: SD rats were treated with BSZY-D for cerebrospinal fluid (CSF) extraction. GT1-7 cells were subjected to different treatments. The phosphorylation levels of JAK2 and STAT5, and the expressions of PRLR and kisspeptin of GT1-7 cells in different groups were detected by western blot, RT-qPCR and immunofluorescence. The expressions of CSN5 and GATA1 and other molecular features were checked by western blot, RT-PCR, coimmunoprecipitation and renilla luciferase activity.

Results: The phosphorylation levels of JAK2 and STAT5, and the expressions of PRLR and kisspeptin in the HPRL group were significantly decreased, and these changes could be reversed after BSZY-D treatment. In addition, the presence of PRLR deubiquitination was detected in the HPRL group, which could be reversed by shRNACSN5, suggesting that BSZY-D played a role through targeting CSN5. The binding level of GATA1 and CSN5 promoter in the HPRL group was significantly decreased, but elevated in the HPRL (BSZY-D/CSF) group (P < 0.05).

Conclusion: BSZY-D improved the transcription activity of GATA1 and increased the binding of GATA1 and CSN5. BSZY-D was involved in the deubiquitination of PRLR, which contributes to alleviating the symptoms of HPRL infertility.

\section{INTRODUCTION}

Hyperprolactinemia (HPRL) is caused by a variety of factors and characterized by the significant elevation of serum prolactin (PRL). It is a syndrome of reproductive-endocrine disorder in the hypothalamicpituitary axis, with relatively high morbidity [1]. At present, although there is no consistent detection method or reference range among different hospitals, it is widely accepted that the subject with serum PRL value more than $1.4 \mathrm{nmol} / \mathrm{L}$ should be diagnosed as HPRL [2]. The pathogenesis of HPRL includes the physiological, pathological, and pharmacological factors [3], among which the physiological factors include diet, sleep, gestation, nipple stimulation and breastfeeding; common pathological factors mainly include hypothalamic tumors, pituitary or ectopic prolactinoma, and acromegaly; as for pharmacological factors, some anesthetic (cocaine) and Chinese herbal medication (Angong Niuhuang Pill) can induce the 
HPRL. The main clinical symptoms include galactorrhea, menstrual disorders, hirsutism, and even infertility [4]. Nowadays, the medication such as bromocriptine is mostly applied in clinical practice. Although the application of bromocriptine has a certain curative effect, the side effects such as dizziness, nausea, vomiting, and resistance to dopamine receptor agonists cannot be ignored. Therefore, it is necessary to explore more safe and effective therapeutic strategies for patients with HPRL infertility.

The prescription of Bu-Shen-Zhu-Yun decoction (BSZY-D) created by Professor Guicheng Xia, is composed of yam, vinegar bupleurum, antlers tablets, red white peony root, cornus, dodder, salvia, angelica, amethyst, poria, fried cortex moutan, etc. This decoction has been proved to regulate menstruation and assist pregnancy [5]. BSZY-D has been widely applied in gynecological diseases, such as luteal phase defect, anovulatory infertility, and polycystic ovarian syndrome [5-7]. However, the efficacy of BSZY-D in HPRL infertility remains elusive.

Kisspeptin, a polypeptide hormone encoded by KISS-1 gene, can regulate the female reproductive endocrine functions. Kisspeptin modulates the secretion of gonadotropin-releasing hormone $(\mathrm{GnRH})$ through the feedback effects of estradiol, and subsequently is involved in the regulation of reproductive and growth hormones in the reproductive system [8]. Hence, kisspeptin contributes to maintaining the stability of the hypothalamus-pituitary-ovarian (HPO) axis. A previous study has reported that BSZY-D can regulate the secretion of serum progesterone, follicle-stimulating hormone, luteinizing hormone, and estradiol through kisspeptin/GPR54 pathway, thereby improving luteal function and promoting embryo implantation [9].

PRL binds to its receptor PRLR and forms a complex that can further activate the Janus kinase 2 (JAK2). Subsequently, the activated JAK2 leads to its downstream signal transducer and activator of transcription 5 (STAT5) [10]. Phosphorylated STAT5 protein forms homodimers or heterodimerizes with other STAT family members, relating to the nucleus translocation and transactivation of the molecular target. JAK2/STAT5 signaling pathway is involved in the development of HPRL, and PRL/PRLR-JAK2/STAT5 signaling pathway is widely explored in cell proliferation, differentiation, and immune regulation. It can also initiate the transcription of certain target genes, and enable the expressions of ovarian and endometrial related receptors.

High PRL level elevates the combination of PRL and PRLR. On the one hand, it can activate the downstream
JAK2/STAT5 pathway in a short time. On the other hand, it can lead to the ubiquitination, endocytosis, and degradation of PRLR, causing inhibition of PRLmediated JAK2/STAT5 pathway in a long period. Excessive PRL cannot bind to its receptor, thus the expressions of JAK2/STAT5 pathway and its downstream kisspeptin are suppressed. CSN5, a type of deubiquitinating enzymes, can inhibit the degradation of PRLR through deubiquitination. We analyzed the transcription factors of CSN5 in the GENERADAR website (https://www.gcbi.com.cn/gcanalyze/html/gene radar/index), and found that GATA1 is one of the transcription factors of CSN5. Hence, the improvement of the deubiquitination level of PRLR in patients with HPRL is the key for the treatment of infertility, miscarriage, irregular menstruation and so on. Therefore, the illustration of BSZY-D underlying mechanism in HPRL is the crucial part. However, no research has reported the relationship between PRLR deubiquitination and BSZY-D.

In the current study, we hypothesized that BSZY-D could improve the HPRL infertility through PRLR deubiquitination, targeting JAK2/STAT5 pathway and its downstream kisspeptin of hypothalamus. Our purpose is to explore the underlying molecular mechanism of HPRL infertility, thereby providing the new theoretical basis for the potential treatment application in clinic.

\section{RESULTS}

\section{Effect of PRL on PRLR/JAK2/STAT5 pathway in GT1-7}

The viability of GT1-7 cells under different concentrations of PRL treatment was detected, and the results showed that PRL (400 ng/ml) has a significant inhibitory effect on the viability of GT1-7 cells, compared with the control group $(\mathrm{P}<0.05)$. No effects have been found in any concentration of CFS on the viability of GT1-7 cells (Supplementary Figure 2). Hence, we used PRL at the concentration of $0 \mathrm{ng} / \mathrm{mL}$, $12.5 \mathrm{ng} / \mathrm{mL}, 25 \mathrm{ng} / \mathrm{mL}, 50 \mathrm{ng} / \mathrm{mL}, 100 \mathrm{ng} / \mathrm{mL}, 200$ $\mathrm{ng} / \mathrm{mL}$, and CFS at a concentration of $10 \%$. Then the phosphorylation levels of JAK, STAT5, and the levels of PRLR and kisspeptin of GT1-7 cells in different groups were detected by western blot (Figure 1). Our data represented that compared with the Control group, the phosphorylation levels of JAK2 and STAT5, and the levels of PRLR and kisspeptin in the PRL $(100 \mathrm{ng} / \mathrm{ml}$ and $200 \mathrm{ng} / \mathrm{ml}$ ) group were significantly decreased (P < 0.05). Consistently, the mRNA expression of kisspeptin was significantly declined in the PRL $(100 \mathrm{ng} / \mathrm{ml}$ and $200 \mathrm{ng} / \mathrm{ml}$ ) group, but no apparent change was detected in PRLR mRNA expression (Supplementary Figure 3). 
Therefore, PRL was concentration-dependent. The higher PRL had a more significant effect, so we took $200 \mathrm{ng} / \mathrm{mL}$ PRL for the subsequent experiments.

\section{The regulation of BSZY-D on PRLR/JAK2/STAT5 pathway via CSF in GT1-7}

The phosphorylation levels of JAK, STAT5, and the levels of PRLR and kisspeptin in different groups were detected by western blot (Figure 2). Our data indicated that compared with the Control group, the phosphorylation levels of JAK2 and STAT5, and the levels of PRLR and kisspeptin in the HPRL group were decreased significantly $(\mathrm{P}<0.05)$. Compared with the HPRL group, the phosphorylation levels of AK2 and STAT5, and the levels of PRLR and kisspeptin in the HPRL (BSZY-D/CSF) group were significantly elevated $(\mathrm{P}<0.05)$, indicating that BSZY-D reversed the impact of HPRL on the downregulation of the PRLR/JAK/STAT5 pathway.

Similarly, the kisspeptin mRNA expression in the HPRL group was significantly decreased, however, the PRLR mRNA expression was not downregulated significantly; compared with the HPRL group, the kisspeptin mRNA expression in the HPRL (BSZY$\mathrm{D} / \mathrm{CSF}$ ) group was significantly increased, suggesting that BSZY-D reversed the downregulation trend of kisspeptin through HPRL (Supplementary Figure 4).

\section{The changes of PRLR expression in GT1-7 cells treated with different reagents}

The level of PRLR in different GT1-7 cell groups were detected by western blot assay. As shown in Figure 3, compared with the Control group, the cytomembrane PRLR level in the HPRL group was significantly inhibited, but the total protein level of PRLR remained stable. Compared with the HPRL group, the protein level of PRLR in the HPRL (BSZY-D/CSF) group was significantly upregulated, which suggested that BSZY-D reversed the downregulation of PRLR protein level via HPRL (P < 0.05). Besides, we found that the PRLR ubiquitination level was increased in the HPRL group, however, there was deubiquitination of PRLR in the HPRL (BSZY-D/CSF) group $(\mathrm{P}<0.05)$. It was indicated that HPRL caused the PRLR ubiquitination, but BSZY-D induced the deubiquitination of PRLR (Supplementary Figure 5).

Compared with the Control group, the PRLR protein level was downregulated in the HPRL group, but there was no difference in total protein level. Moreover,
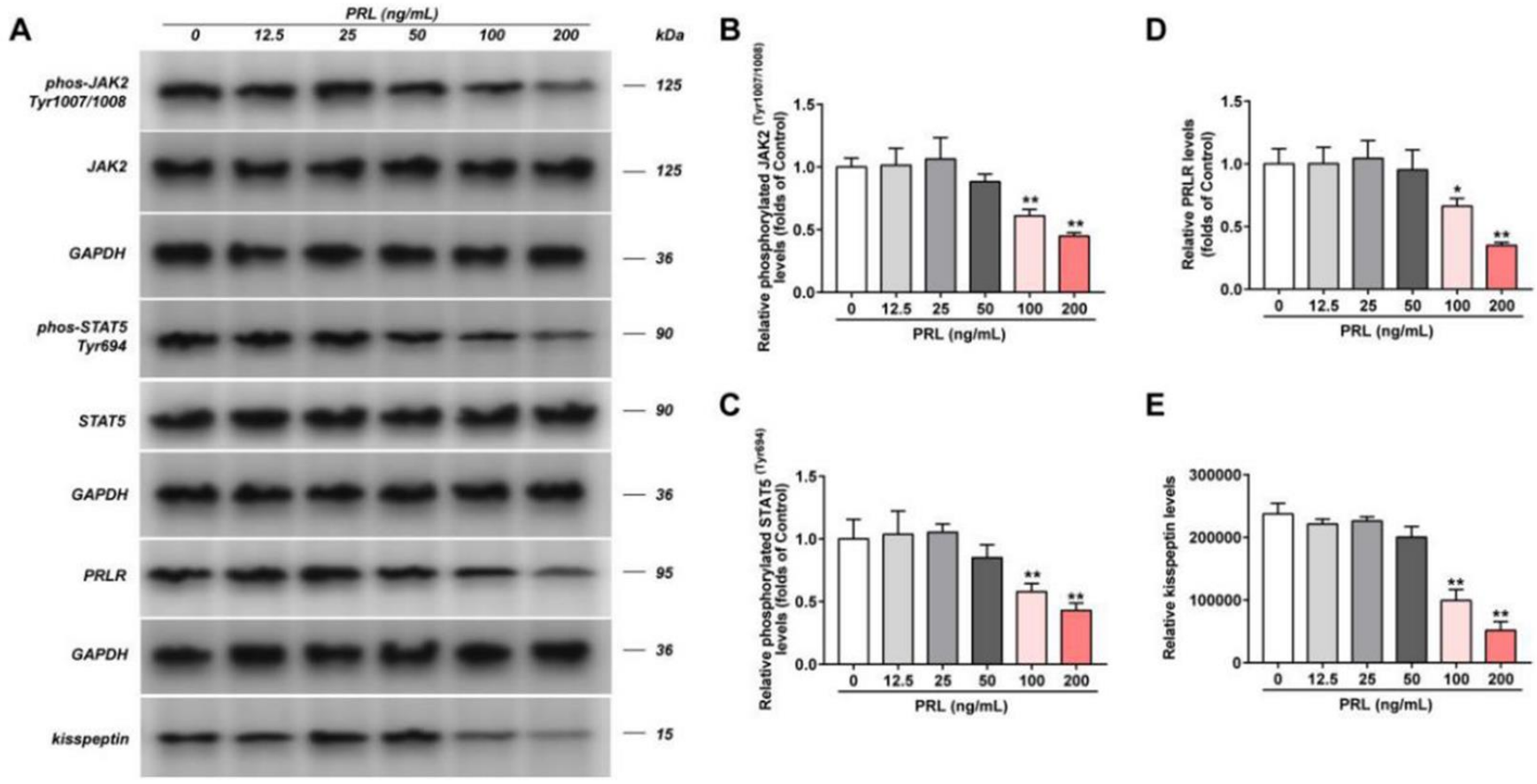

Figure 1. Effect of PRL on PRLR-JAK-STAT5 pathway in GT1-7. The phosphorylation levels of JAK, STAT5 and the levels of PRLR and kisspeptin in GT1-7 from different groups were detected by western blot assay, and representative bands were shown in (A). The phosphorylation levels of JAK (B) and STAT5 (C), and the level of PRLR (D), and the level of kisspeptin (E) were normalized to control. The results were presented as mean $\pm S D(n=3) .{ }^{*} p<0.05,{ }^{* *} p<0.01$ vs. Control group. 
A

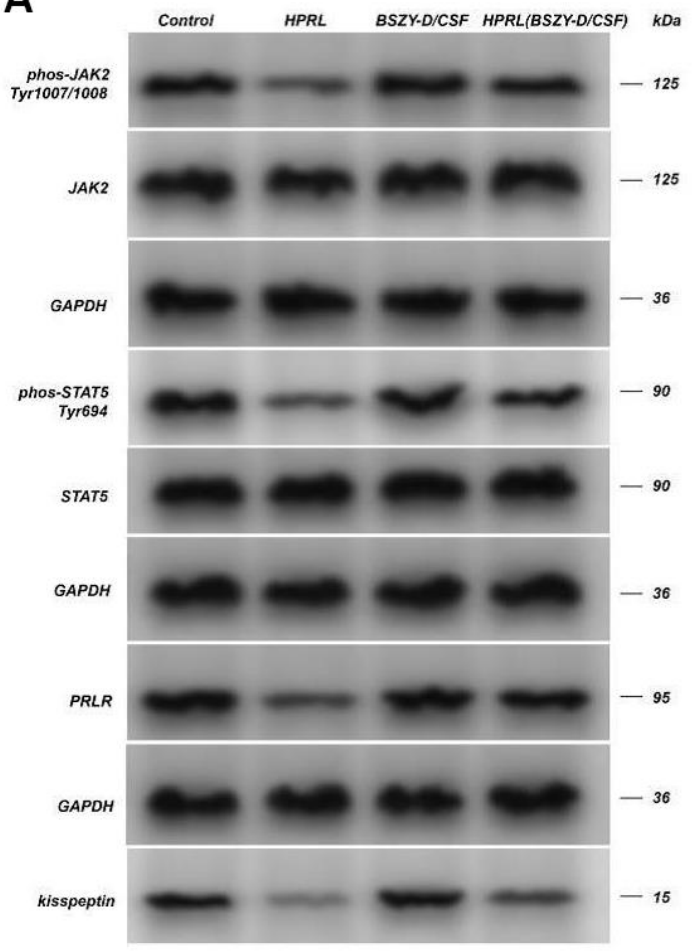

B

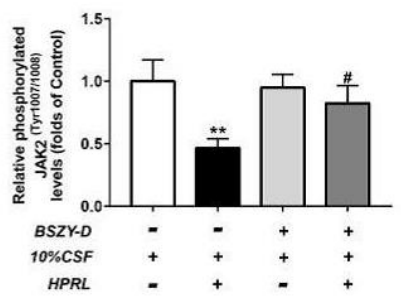

C

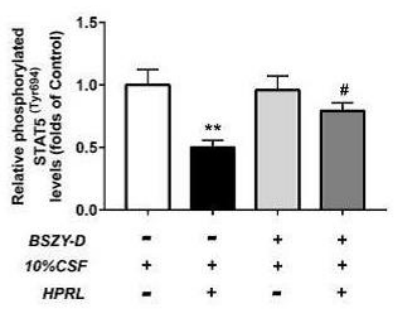

D

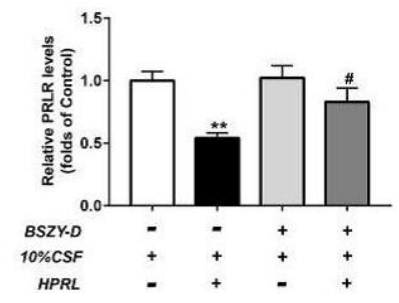

E

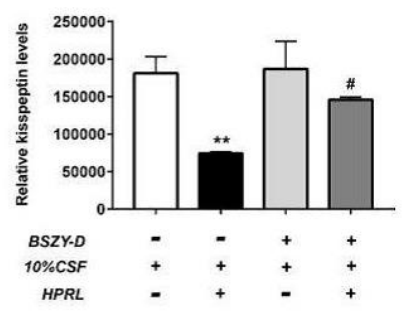

Figure 2. Effect of CSF on PRLR-JAK-STAT5 pathway in GT1-7. The phosphorylation levels of JAK, STAT5 and the levels of PRLR and kisspeptin in GT1-7 from different groups were detected by western blot assay, and representative bands were shown in (A). The phosphorylation levels of JAK (B) and STAT5 (C), and the level of PRLR (D), and the level of kisspeptin (E) were normalized to control. The results were presented as mean $\pm S D(n=3) . * * p<0.01$ vs. Control group, $\# p<0.05, \# \# p<0.01$ vs. HPRL group.
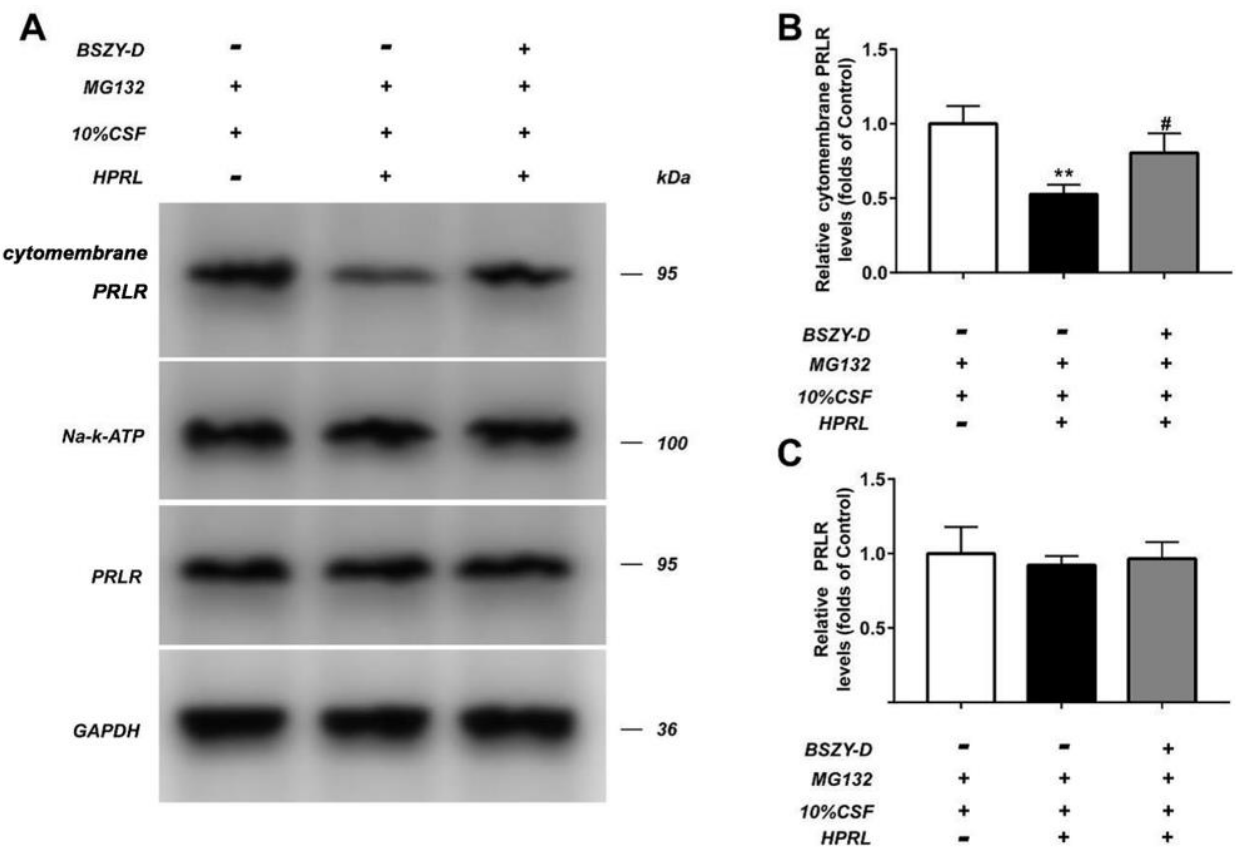

Figure 3. The level of PRLR in GT1-7 from different groups was detected by western blot assay, and representative bands were shown in (A). The levels of PRLR (B, C) were normalized to control. The results were presented as mean $\pm \operatorname{SD}(n=3) . * * p<0.01$ vs. Control group, \#p $<0.05$, $\#$ \# $<0.01$ vs. HPRL group. 
compared with the HPRL group, the PRLR protein level was augmented in the HPRL (BSZY-D/CSF) group, suggesting that CSF reversed the downregulation of PRLR protein level through HPRL $(\mathrm{P}<0.05)$ (Figure 4).

The PRLR protein level of GT1-7 cells at different time points were detected using western blot. Compared with the Control + CHX group, the PRLR protein level was decreased significantly in the HPRL group. Compared with the HPRL + CHX group, the PRLR level in the HPRL (BSZY-D/CSF) + CHX group was significantly upregulated $(\mathrm{P}<0.05)$. The results demonstrated that the PRLR degradation would be accelerated under the high PRL condition, and this degradation was inhibited after the addition of BSZY-D (Figure 5).

\section{The changes of CSN5 expression in GT1-7 cells treated with different reagents}

Co-IP was applied to test the expressions of $\beta$-Trcp, PRLR and CSN5 (Supplementary Figure 6). Compared with the Control group, the binding of PRLR and $\beta$ Trcp was significantly increased in the HPRL group, and the binding of PRLR and CSN5 was decreased; compared with the HPRL group, the binding of PRLR and CSN5 in the HPRL (BSZY-D/CSF) group was increased $(\mathrm{P}<0.05)$. It was suggested that BSZY-D played a role by promoting the binding of PRLR and CSN5.

The levels of CSN5 and GATA1 in GT1-7 were detected by western blot (Figure 6). Our results represented that the CSN5 and GATA1 protein levels were significantly decreased in the HPRL group compared with the Control group. Compared with the HPRL group, the levels of CSN5 and GATA1 in the HPRL (BSZY-D/CSF) group were significantly elevated $(\mathrm{P}<0.05)$. In line with the results of western blot, the RT-PCR data showed that the CSN5 mRNA expression was significantly suppressed in the HPRL group compared with the Control group, and the GATA1 mRNA expression did not change significantly. Besides, compared with the HPRL group, the CSN5

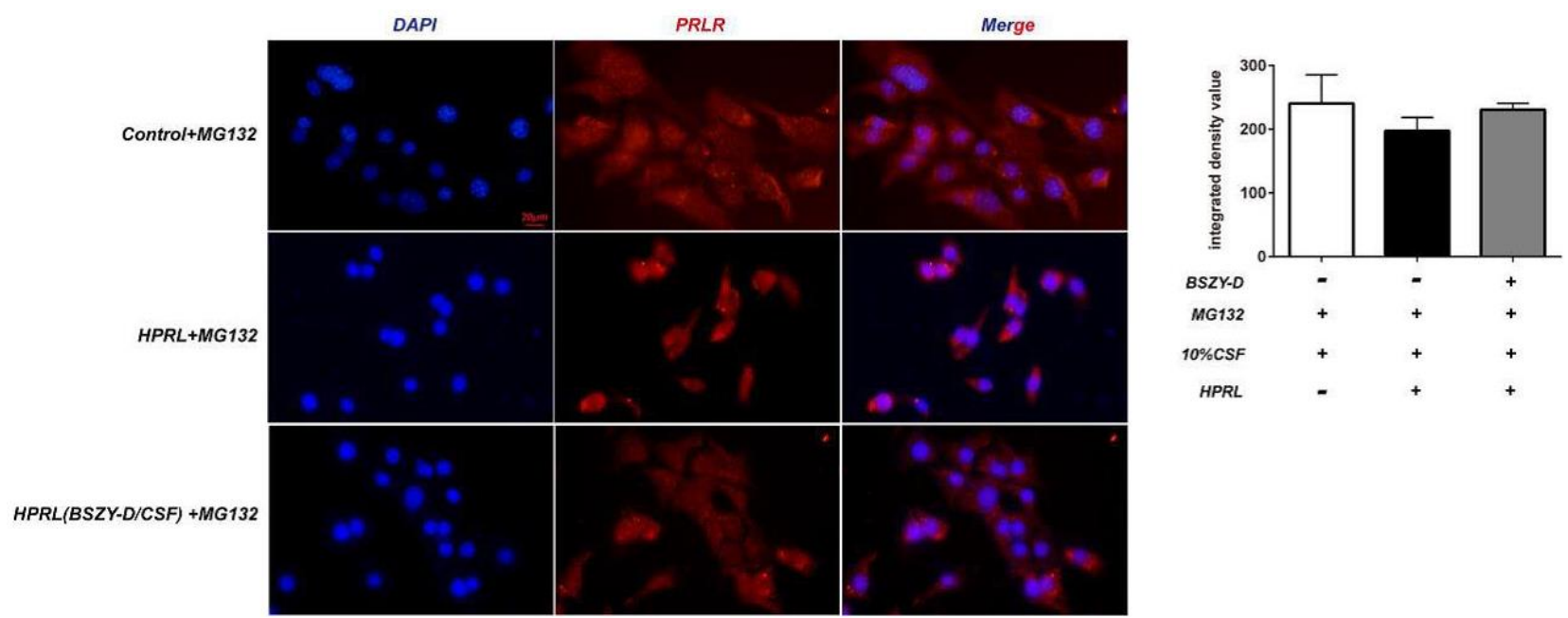

Figure 4. The immunofluorescence staining of PRLR in GT1-7.
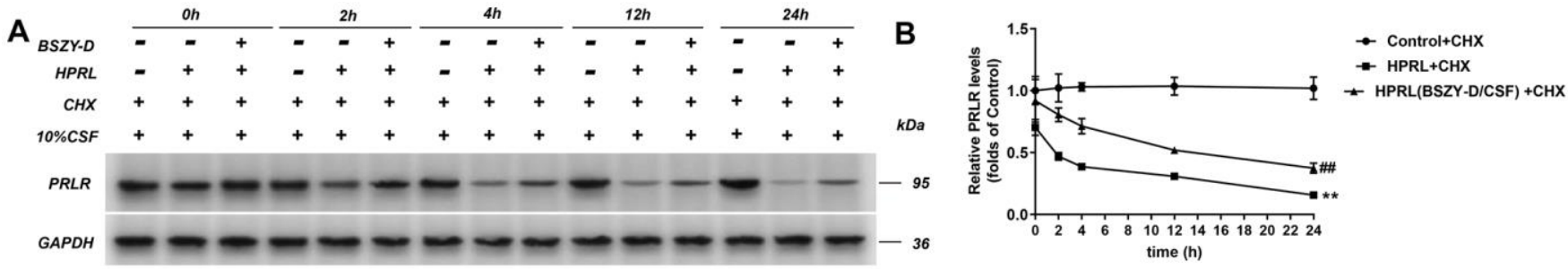

Figure 5. The level of PRLR in GT1-7 at different times was detected by western blot assay. The levels of PRLR were normalized to control. (A) Representative blot of PRLR level. (B) Quantitative relative gray values of PRLR protein level. The results were presented as mean $\pm S D(n=3) .{ }^{* *} p<0.01$ vs. Control group, $\# \# p<0.01$ vs. HPRL group. 
mRNA expression was significantly increased in the HPRL (BSZY-D/CSF) group $(\mathrm{P}<0.05)$ (Supplementary Figure 7). It was indicated that BSZY-D reversed the downregulation effect of HPRL on CSN5.

\section{Effect of lentivirus plasmids shRNA-CSN5 on PRLR/JAK/STAT5 pathway in GT1-7}

Luciferase activity was detected by measuring the renilla luciferase activity of GT1-7 cells transiently transfected with the CSN5 luciferase promoter (Figure 7). Compared with the Control group, the CSN5 promoter activity in the HPRL group was significantly decreased; in turn, compared with the HPRL group, the CSN5 promoter activity in the HPRL (BSZY-D/CSF) group was significantly upregulated. Correspondingly, the binding of GATA1 and CSN5 promoter in the HPRL group was significantly decreased, but the binding of GATA1 and CSN5 promoter in the HPRL (BSZY-D/CSF) group was significantly elevated (P < 0.05) (Supplementary Figure 8). After the transfection of lentivirus plasmids, the CSN5 protein level was significantly inhibited in the shRNA-CSN5 group, suggesting that the shRNA-CSN5 plasmid reduced the expression of CSN5 in GT1-7 cells $(\mathrm{P}<0.05)$ (Supplementary Figure 9).

A

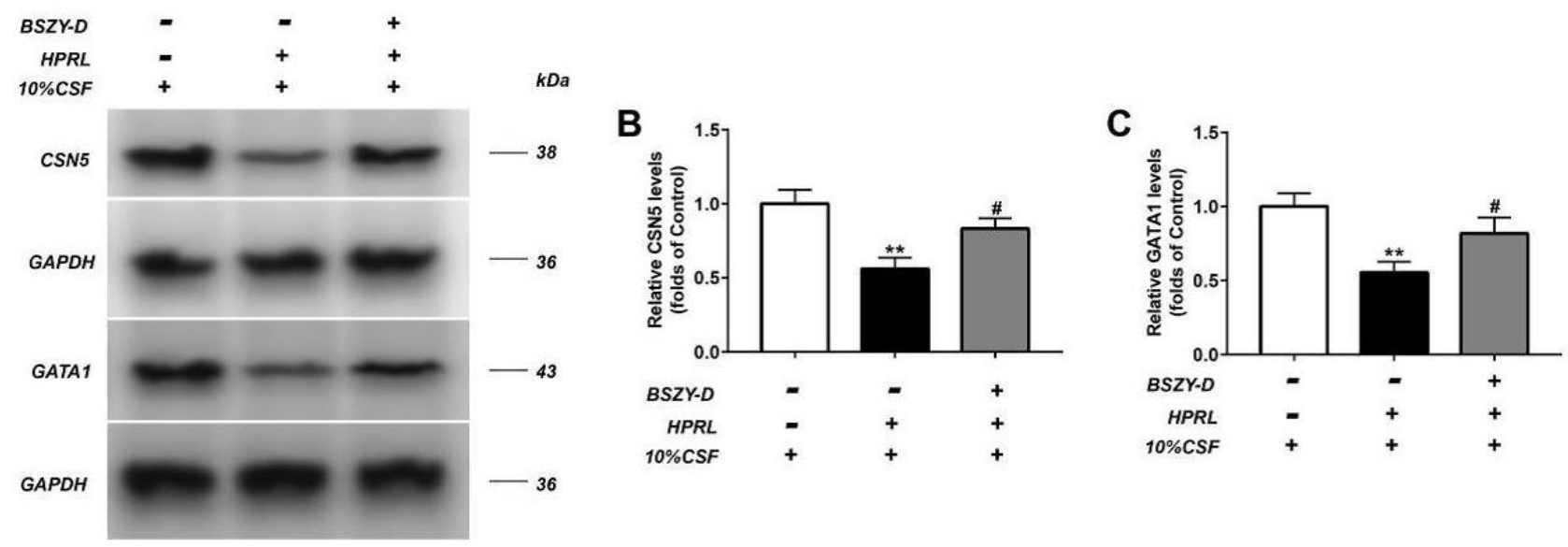

Figure 6. The levels of CSN5 and GATA1 in GT1-7 were detected by western blot assay, and representative bands were shown in (A). The levels of CSN5 (B) and GATA1 (C) were normalized to control. The results were presented as mean \pm SD $(n=3)$. ${ }^{* *} p<0.01$ vs. Control group, $\# p<0.05, \# \# p<0.01$ vs. HPRL group.

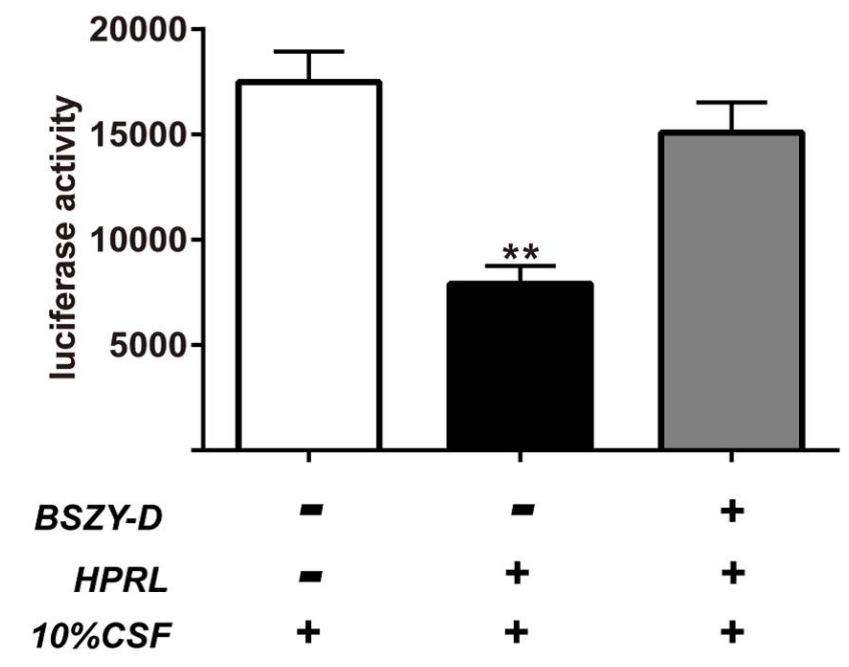

Figure 7. Luciferase activity measured and normalized according to renilla luciferase activity in GT1-7 cells transiently transfected with the CSN5 luciferase promoter. The results were presented as mean \pm SD $(n=3)$. ${ }^{* *} p<0.01$ vs. Control group, \#\#p $<$ 0.01 vs. HPRL group. 
After the confirmation of transfection efficiency, the impact of shRNA-CSN5 on PRLR/JAK/STAT5 signaling pathway in GT1-7 cells of different treatment groups was detected by western blot. As shown in Figure 8, compared with the Control group, the phosphorylation levels of JAK2 and STAT5, and the protein levels PRLR and kisspeptin in the HPRL (BSZY-D/CSF) + shRNA-NC group were significantly decreased $(\mathrm{P}<0.05)$. After knocking down CSN5 gene, the above-mentioned protein levels could be reversed. This result indicated that BSZY-D enhanced the PRLR/JAK/STAT5 signaling pathway through CSN5.
Besides, there existed an increasing ubiquitination level of PRLR in the HPRL group, which could be reversed by knocking down shRNA-SCN5. It was suggested that BSZY-D played a role through targeting CSN5 (Supplementary Figure 10).

\section{DISCUSSION}

Previous researches of traditional Chinese medicine $\mathrm{Bu}$ Shen-Zhu-Yun Decoction (BSZY-D) mainly focus on infertility caused by luteal phase defect. Recently, it is reported that BSZY-D can reduce the prolactin (PRL)

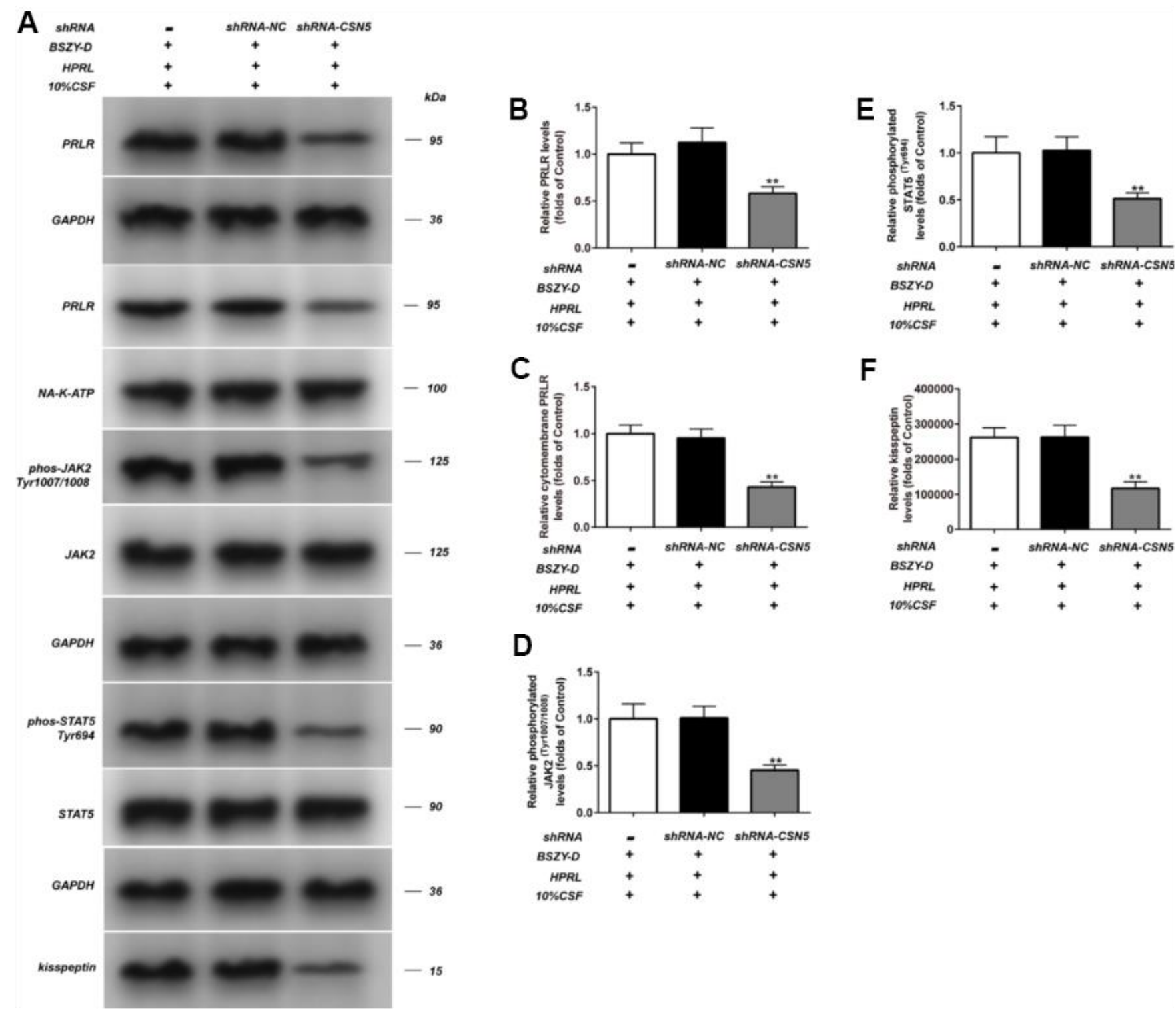

Figure 8. Effect of shRNA-CSN5 on PRLR-JAK-STAT5 pathway in GT1-7. The phosphorylation levels of JAK, STAT5 and the levels of PRLR and kisspeptin in GT1-7 from different groups were detected by western blot assay, and representative bands were shown in (A). The phosphorylation levels of JAK (D) and STAT5 (E), and the level of PRLR (B, C), and the level of kisspeptin (F) were normalized to control. The results were presented as mean $\pm S D(n=3)$. ${ }^{*} p<0.05,{ }^{* *} p<0.01$ vs. Control group. 
level in patients with luteal phase defect and hyperprolactinemia (HPRL) in clinic practice. Hence, our purpose in this study is to investigate the underlying mechanism of BSZY-D in decreasing PRL. In this study, we identified that HPRL could downregulate the levels of PRLR and kisspeptin, and phosphorylation levels of JAK2 and STAT5. BSZY-D reversed the effect of HPRL on the downregulation of the PRLR/ JAK2/STAT5 pathway. The potential mechanism was that excessive PRL cannot bind to its receptor under high PRL microenvironment, thus the expressions of JAK2/STAT5 pathway and kisspeptin were suppressed. Besides, CSN5 inhibited the degradation of PRLR through deubiquitination, and targeted its transcription factors GATA 1 . These changes could be reversed after BSZY-D treatment. We proved that BSZY-D increased the binding level of CSN5 and GATA 1, and induced the deubiquitination of PRLR, thus increasing the expressions of JAK2/STAT5 pathway and its downstream signaling kisspeptin. Based on our study, BSZY-D can be regarded as an effective Chinese medicine to treat HPRL infertility in clinic (Figure 9).

The theory of Chinese medicine believes that the kidney affects reproduction, which is the innate foundation and the root of vitality. Ovulation relies on several factors, such as kidney, liver, spleen, qi, blood, yin and yang [7]. A previous study of meta-analysis has represented that BSZY-D can significantly elevate the pregnancy rate to $50 \%$, comparing to the pregnancy rate of $30 \%$ using vitro fertilization [11]. Several ingredients of BSZY-D including dioscoreae rhizome, cervi cornu, bupleuri radix and paeoniae radix alba, etc. have been proved to improve the uterine receptivity and pregnancy rate in mice $[12,13]$. Xiaofei Jiang et al. have demonstrated that BSZY-D can upregulate the expressions of integrin $\alpha 5$ and $\beta 3$ by inhibiting the estrogen and progesterone receptor, meanwhile, improve the endometrial receptivity during embryo implantation and consequently enhance the pregnancy rates [14]. In the current study, we found that BSZY-D reserved the molecular and pathway changes caused by HPRL. The elevation of PRLR, kisspeptin, phosphorylated JAK2, and STAT5 levels were significantly reserved by BSZY-D treatment, as well as the decline of the PRLR ubiquitination.

Prolactin (PRL) is a type of polypeptide hormone secreted by the anterior pituitary gland. PRL is balanced by PRL releasing factor (PRF) and PRL inhibiting hormone (PIH) $[15,16]$. In the normal physiological condition, the increased transcription activity of GATA1 induces the transcription of its downstream CSN5 gene, leading to the upregulation of CSN5. CSN5 plays a role in mediating the deubiquitination of PRLR, blocking its endocytosis and degradation, and maintaining the normal function of PRLR. JAK2/STAT5 is the downstream signaling pathway of PRLR, and the activation of JAK2/STAT5 can elevate the expression of kisspeptin. In the presence of pathological HPRL condition, HPRL can reduce the

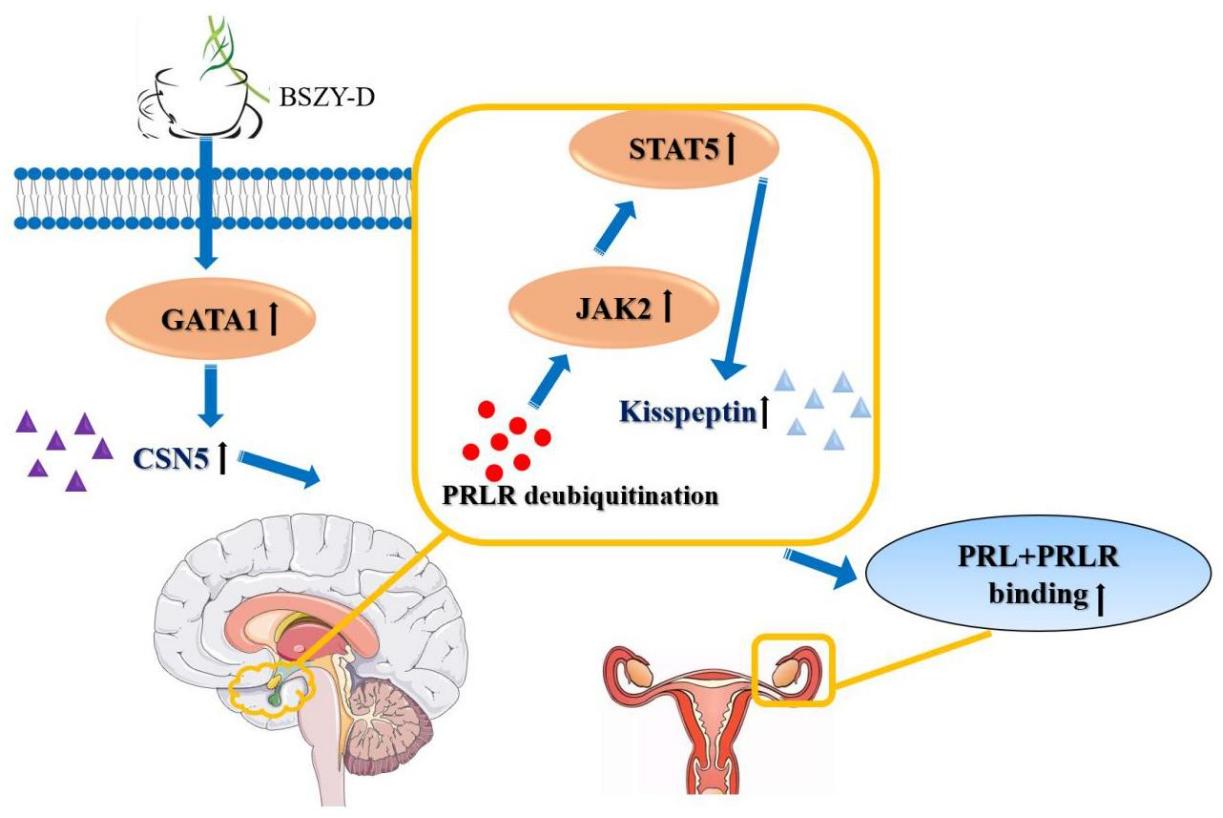

Figure 9. The effects of BSZY-D on activating GATA 1 and CSN5, inducing PRLR deubiquitination and increasing the expressions of JAK2/STAT5 signaling pathway and kisspeptin. Hence, BSZY-D had an efficient role in HPRL infertility improvement. 
receptivity of the endometrium, and suppress the ovarian function, consequently causing ovulation disorders [17]. Recently, it is reported that the proper concentration of PRL can promote the growth of endometrial cells and blastocyst adhesion. However, the deregulated PRL concentration has an inhibitory effect, which causes follicle maturation disorder, ovulation disorder and decreased endometrial receptivity, thus eventually leading to infertility [18]. The underlying mechanism can be explained by an abnormal level of PRLR ubiquitination. The overexpressing PRL augments the activity of CSN5, a type of deubiquitinating enzyme, which induces the deubiquitination of PRLR. Hence, the downstream JAK2/STAT5 signaling pathway is inhibited, as well as kisspeptin. In the current study, we identified that BSZY-D could reversely elevate the transcription activity of GATA1, increase the binding of GATA1 and CSN5, and then reserve the downstream expressions at a certain level.

Kisspeptin is a type of polypeptide encoded by the KISS-1 gene. It is mainly distributed in the hypothalamus, pituitary, ovary, placenta, etc. Recent studies have represented that kisspeptin plays an important role in the initiation of puberty, menstrual cycle regulation, ovulation induction, and reproductive cycle regulation [19]. Some scholars have indicated that kisspeptin may have a certain correlation with pregnancy implantation, and further hypothesized it is also related to abortion [20]. In the current study, our results showed that HPRL could downregulate the expression of kisspeptin, which might impair the physiological role of kisspeptin in maintaining the normal productive function. Interestingly, kisspeptin expression could be reversed after BSZY-D treatment. Besides, in line with the changes of kisspeptin, the expression of PRLR/JAK/STAT5 signaling pathway showed the similar trend.

In this study, we proved that BSZY-D partly recovered the expressions of JAK/STAT5 and kisspeptin via inducing the deubiquitination of PRLR and targeting CSN5. The limitation of this study is that we did not clarify the possible molecular mechanism between CSN5 and PRLR deubiquitination, which will be investigated as a key point in our further study.

\section{CONCLUSIONS}

Altogether, we explored the underlying mechanism of BSZY-D in HPRL infertility. We demonstrated that BSZY-D could improve the transcription activity of GATA1 and increase the binding of GATA1 and CSN5. Also, the activated deubiquitinating enzyme CSN5 could increase the expressions of JAK/STAT5 and kisspeptin via inducing the deubiquitination of PRLR, thereby alleviating the clinic manifestations of HPRL infertility. We hope our novel work could establish a theoretical basis on the molecular mechanism of BSZY$D$ in HPRL infertility, meriting further investigation of potential treatment application.

\section{MATERIALS AND METHODS}

\section{Cell culture and treatment}

GT1-7 cell line was purchased from Millipore (Schwalbach, Germany). GT1-7 cells were cultured and maintained in DMEM medium (GIBCO, Grand Island, USA) containing penicillin (final concentration $100 \mathrm{U} / \mathrm{mL}$ ) (Sigma-Aldrich, St. Louis, MO, USA), streptomycin (final concentration $100 \mu \mathrm{g} / \mathrm{mL}$ ) (SigmaAldrich, St. Louis, MO, USA), 2mM L-Glutamine (Millipore, Schwalbach, Germany) and 10\% fetal bovine serum (FBS) (Hyclone, Logan, UT, USA). The cells were digested when reaching $90 \%$ confluence.

\section{Medicine and extraction preparation}

BSZY-D was concentrated to $2 \mathrm{~g} / \mathrm{mL}$ and stored at $4^{\circ} \mathrm{C}$. In brief, the medicinal materials had been immersed in water for $30 \mathrm{~min}$. Firstly, the deer horn slices were decocted and boiled for $20 \mathrm{~min}$, and then added with the remaining medicinal materials. The decoction at a concentration of $2 \mathrm{~g} / \mathrm{mL}$ was collected and stored at $4^{\circ} \mathrm{C}$.

A total of 75 female SD rats of specific pathogen-free (SPF) grade (aged 8 weeks and weighing $220 \pm 20$ g) were purchased from Animal Model Center of Nanjing University [license No: SCXK (Su) 20180008], and raised in the SPF environment in the Experimental Animal Center of Nanjing Chinese Medicine University. The rats were reared at $40 \%$ $70 \%$ humidity and $21-25^{\circ} \mathrm{C}$, maintained in a $12 \mathrm{~h}$ light/dark cycle. The experimental operations were approved by the Animal Ethics Committee of Nanjing Chinese Medicine University [approval No: SYXK (Su) 2014-0001].

Cerebrospinal fluid was prepared as follows: BSZY-D of $2 \mathrm{~g} / \mathrm{mL}$ was given to normal female SD rats $1 \mathrm{~mL} / \mathrm{kg}$ twice a day. After anesthesia, the neck skin of each rat was disinfected with $75 \%$ ethanol, and the foramen magnum was exposed. The micro-injector was pierced into the cistern magna and $100 \mathrm{uL}$ cerebrospinal fluid (CSF) was slowly extracted. After centrifugation at $2000 \mathrm{r} / \mathrm{min}$ for $15 \mathrm{~min}$, the supernatant was collected and stored at $-20^{\circ} \mathrm{C}$. The time point of CSF collection was after the estrus. 


\section{Experimental groups}

Protocols in different experimental groups were shown as follows:

1) Experiment 1: 0\% CSF (medicated cerebrospinal fluid), 5\% CSF (medicated cerebrospinal fluid), $10 \%$ CSF (medicated cerebrospinal fluid) and $20 \% \mathrm{CSF}$ (medicated cerebrospinal fluid).

2) Experiment 2: $0 \mathrm{ng} / \mathrm{mL}, 12.5 \mathrm{ng} / \mathrm{mL}, 25 \mathrm{ng} / \mathrm{mL}, 50$ $\mathrm{ng} / \mathrm{mL}, 100 \mathrm{ng} / \mathrm{mL}, 200 \mathrm{ng} / \mathrm{mL}$ and $400 \mathrm{ng} / \mathrm{mL}$ PRL.

3) Experiment 3: $0 \mathrm{ng} / \mathrm{mL}, 12.5 \mathrm{ng} / \mathrm{mL}, 25 \mathrm{ng} / \mathrm{mL}, 50$ $\mathrm{ng} / \mathrm{mL}, 100 \mathrm{ng} / \mathrm{mL}$ and $200 \mathrm{ng} / \mathrm{mL}$ PRL.

4) Experiment 4:

Normal group (Control group): $10 \%$ blank cerebrospinal fluid, cultured for $72 \mathrm{~h}$.

PRL intervention group (HPRL group): $10 \%$ blank cerebrospinal fluid $+200 \mathrm{ng} / \mathrm{mL}$ PRL, cultured for $72 \mathrm{~h}$.

Cerebrospinal fluid containing BSZY-D intervention group (BSZY-D/CSF group): 10\% BSZY-D containing medicated cerebrospinal fluid, cultured for $72 \mathrm{~h}$.

Cerebrospinal fluid containing traditional Chinese medicine at a concentration of $10 \%$ [HPRL (BSZYD/CSF) group]: $10 \%$ BSZY-D containing cerebrospinal fluid culture $+10 \%$ blank cerebrospinal fluid +200 $\mathrm{ng} / \mathrm{mL}$ PRL, cultured for $72 \mathrm{~h}$.

\section{5) Experiment 5:}

Normal group + MG132 (Selleck Chemicals, Houston, Texas, USA) (Control + MG132 group): 10\% blank cerebrospinal fluid + 10 uM MG132 inhibitor, cultured for $72 \mathrm{~h}$.

PRL intervention group + MG132 (HPRL + MG132 group): $10 \%$ blank cerebrospinal fluid $+200 \mathrm{ng} / \mathrm{mL}$ PRL + 10 uM MG132 inhibitor, cultured for $72 \mathrm{~h}$.

Cerebrospinal fluid containing traditional Chinese medicine a concentration of $10 \%+$ MG132 [HPRL (BSZY-D/CSF) group]: 10\% BSZY-D containing cerebrospinal fluid culture $+200 \mathrm{ng} / \mathrm{mL}$ PRL $+10 \mathrm{uM}$ MG132 inhibitor, cultured for $72 \mathrm{~h}$.

6) Experiment 6:

Normal group + cycloheximide (CHX) (Seleck Chem, Shanghai, China) (Control + CHX group): 10\% blank cerebrospinal fluid $+10 \mathrm{uM} \mathrm{CHX}$ inhibitor.
PRL intervention group + cycloheximide CHX (HPRL + CHX group): $10 \%$ blank cerebrospinal fluid +200 ng/mL PRL + 10uM CHX inhibitor.

Cerebrospinal fluid containing traditional Chinese medicine at a concentration of $10 \%+$ cycloheximide CHX [HPRL (BSZY-D/CSF) + CHX group]: $10 \%$ BSZY-D containing medicated cerebrospinal fluid culture $+200 \mathrm{ng} / \mathrm{mL}$ PRL $+10 \mathrm{uM} \mathrm{CHX}$ inhibitor.

The time points for detection were $0 \mathrm{~h}, 2 \mathrm{~h}, 4 \mathrm{~h}, 12 \mathrm{~h}, 24 \mathrm{~h}$, respectively.

7) Experiment 7:

Normal group + MG132 (Control + MG132 group): $10 \%$ blank cerebrospinal fluid +10 uM MG132 inhibitor, cultured for $72 \mathrm{~h}$.

PRL intervention group + MG132 (HPRL + MG132 group): $10 \%$ blank cerebrospinal fluid $+200 \mathrm{ng} / \mathrm{mL}$ PRL + 10 uM MG132 inhibitor, cultured for 72h.

Cerebrospinal fluid containing traditional Chinese medicine at a concentration of 10\% + MG132 [HPRL (BSZY-D/CSF) +MG132 group]: $10 \%$ BSZY-D containing medicated cerebrospinal fluid culture +200 $\mathrm{ng} / \mathrm{mL}$ PRL + $10 \mathrm{uM} \mathrm{MG132}$ inhibitor, cultured for $72 \mathrm{~h}$.

8) Experiment 8:

Normal group (Control group): 10\% blank cerebrospinal fluid, cultured for 72 hours.

Prolactin intervention group (HPRL group): 10\% blank cerebrospinal fluid $+200 \mathrm{ng} / \mathrm{mL}$ PRL, cultured for $72 \mathrm{~h}$.

Cerebrospinal fluid containing traditional Chinese medicine a concentration of $10 \%$ intervention [HPRL (BSZY-D/CSF) group]: 10\% BSZY-D containing medicated cerebrospinal fluid culture $+200 \mathrm{ng} / \mathrm{mL}$ PRL, cultured for $72 \mathrm{~h}$.

9) Experiment 9:

Cerebrospinal fluid containing traditional Chinese medicine at a concentration of $10 \%$ intervention [HPRL (BSZY-D/CSF) group]: 10\% BSZY-D containing medicated cerebrospinal fluid culture $+200 \mathrm{ng} / \mathrm{mL}$ PRL, cultured for $72 \mathrm{~h}$.

Cerebrospinal fluid containing traditional Chinese medicine a concentration of $10 \%+$ ShRNA-CSN5 [HPRL (BSZY-D/CSF) + ShRNA-CSN5 group]: after the cells were stably transfected with CSN5-knockdown 
lentivirus, added 10\% BSZY-D containing medicated cerebrospinal fluid culture $+200 \mathrm{ng} / \mathrm{mL}$ PRL, cultured for $72 \mathrm{~h}$.

Cerebrospinal fluid containing traditional Chinese medicine a concentration of $10 \%+$ ShRNA- NC [HPRL (BSZY-D/CSF) + ShRNA-NC group]: after the cells were stably transfected with NC lentivirus, added $10 \%$ BSZY-D containing medicated cerebrospinal fluid culture $+200 \mathrm{ng} / \mathrm{mL}$ PRL, cultured for $72 \mathrm{~h}$.

\section{CCK8 assay}

The GT1-7 cell density was adjusted to 5000 cells/well, seeded into a 96-well plate, and cultured in the incubator at $37^{\circ} \mathrm{C}$ with $5 \% \mathrm{CO}_{2}$ for $24 \mathrm{~h}$. Afterwards, the original culture solution was discarded and the cells were washed twice with phosphate-buffered saline (PBS). According to Experiment 1 or 2, GT1-7 cells were treated differently. Each experiment group had 3 parallel wells. After $72 \mathrm{~h}$ of incubation, each well was added with $10 \mu \mathrm{L}$ CCK-8 (Beyotime Biotechnology, Shanghai, China) solution and incubated for $2 \mathrm{~h}$ in an incubator. The optical density (OD) value of the liquid in each well was measured by a microreader (Berthold LB941) at the wavelength of $450 \mathrm{~nm}$.

\section{Western blot}

Total protein was extracted from GT1-7 cells using RIPA lysis buffer (Beyotime Biotechnology, Shanghai, China). The tissue lysates were centrifuged at 12,000 rpm for $5 \mathrm{~min}$ at $4^{\circ} \mathrm{C}$, and the supernatants were collected for further analysis. The bicinchoninic acid (BCA) assay was applied to detect the concentration of protein samples. Then, $60 \mu \mathrm{g}$ protein samples per lane were separated using $8 \%$ SDS-PAGE and then transferred onto PVDF membranes $(0.45 \mu \mathrm{m}$, Millipore, Schwalbach, Germany). The membranes were blocked in Tris-buffered saline Tween containing 5\% skim milk for $1 \mathrm{~h}$ at room temperature and then incubated overnight at $4^{\circ} \mathrm{C}$ with primary antibodies: Anti-phosJAK2 (Tyr1007/1008) antibody (3771), Anti-JAK2 antibody (3230), Anti-phos-STAT5 (Tyr694) antibody (9314), Anti-STAT5 antibody (94205), Anti-NA/K ATP antibody (3010) (CST, Boston, USA); Anti-PRLR antibody (ab170935), Anti-kisspeptin antibody (ab19028) (Abcam, Cambridge, UK); Anti-PRLR antibody (ABIN152720) (IP), Anti-GATA1 antibody (ABIN2704828) (antibodies-online, Beijing, China). Next day, these membranes were washed with PBS and incubated with secondary antibodies for $1 \mathrm{~h}$ at room temperature. After rinsing, the proteins were detected by enhanced chemiluminescence. The protein levels were quantified by densitometry using Image-Pro Plus 6.0 and normalized to the corresponding GAPDH level.

\section{Real-time PCR}

The total RNA was extracted from GT1-7 cells using TRIzol reagent (Invitrogen, Carlsbad, CA, USA). RNA then underwent reverse transcription using the iScript cDNA kit (Bio-Rad, Hercules, CA, USA), followed by analysis using real-time PCR (RT-PCR) with the SsoFast Eva Green Super Mix (Bio-Rad, Hercules, CA, USA) on Roche Light Cycler 480 system.

Relative mRNA expressions of kisspeptin, PRLR, CSN5, GATA1, GAPDH were calculated by the comparative cycle threshold (CT) method, with expression of GAPDH as the internal reference. The $2^{-\Delta \Delta \mathrm{Ct}}$ method was used for analysis. The specific primers applied for RT-PCR reaction are shown in Table 1.

\section{Co-immunoprecipitation (Co-IP)}

The GT1-7 cells were treated as described in Experiment 5, 7 and 9. Then the cell precipitation was added with RIPA lysis buffer. Protein A/G-agarose microspheres were washed twice with PBS, and prepared into $50 \%$ protein $\mathrm{A} / \mathrm{G}$-agarose working solution with PBS. Next, 50\% protein A/G agarose working solution was added into the samples at a ratio of $100 \mu \mathrm{L}$ per $1 \mathrm{~mL}$. The protein A/G-agarose microspheres were discarded by centrifugation. BCA assay was applied to test the protein concentration. Afterwards, $5 \mu \mathrm{L}$ antibody (PRLR150) (IgG antibody as the control group) was added for incubation at $4^{\circ} \mathrm{C}$ overnight and the final volume was $500 \mu \mathrm{L}$. Next day, $100 \mu \mathrm{L}$ protein $\mathrm{A} / \mathrm{G}$-agarose microspheres was added to capture the antigen-antibody complex at room temperature for $1 \mathrm{~h}$. The protein A/G-agarose microsphere antigen-antibody complex was collected after centrifugation and washed with PBS. The supernatant was collected, and the loaded samples were boiled for $5 \mathrm{~min}$ to precipitate. The obtained samples were detected by western blot using antibodies ( $\beta$-Trcp, 4394, Abcam, Cambridge, UK), as described in 3.4 .

\section{Immunofluorescence}

The cells were fixed with $10 \%$ paraformaldehyde for 20 min at room temperature. The fixed cells were blocked with $1 \%$ bovine serum albumin (BSA) for $30 \mathrm{~min}$ and incubated with the corresponding primary antibody (PRLR 1:20) at $4^{\circ} \mathrm{C}$ overnight, followed by washing with PBST for $10 \mathrm{~min}$. Then the secondary antibody was added for $2 \mathrm{~h}$-incubation at room temperature, followed by the 4', 6-diamidino-2-phenylindole (DAPI) staining for $15 \mathrm{~min}$, and observation under a laser confocal microscope $(400 \times)$. 
Table 1. Primers used in quantitative real-time PCR and shRNA-CNS5 sequence.

\begin{tabular}{lc}
\hline Primers & Sequences $\left(\mathbf{5}^{\prime} \rightarrow \mathbf{3}^{\prime}\right)$ \\
\hline \multirow{2}{*}{ kisspeptin } & F- CTCTGTGTCGCCACCTATGG \\
& R- AGGCTTGCTCTCTGCATACC \\
PRLR & F- GACTCAAGGGGGCAAAGTCA \\
CSN5 & R- CACCTCCACAGAGAAGCGTT \\
& F- GCCTTGAGAGTCTATCACCACT \\
GATA1 & R- TGATGATCATGGTCTCGCCG \\
& F- TTGGGATCACCCTGAACTCG \\
GAPDH & R- GGTTGAACCTGGGCTTGTTG \\
& F- CATCACTGCCACCCAGAAGACTG \\
ShRNA-CSN5 & R- ATGCCAGTGAGCTTCCCGTTCAG \\
& CCGGAGCGCAGAGTATCGATGAAATCTCG \\
\hline
\end{tabular}

\section{Construction of luciferase reporter gene plasmid}

The full length of CSN5 (NM001277101) promoter was detected, combining with the target gene prediction software (Jaspar), to find the interaction site of CSN5 promoter and acting site of GATA1. Primer 5 was applied to design primers, making PCR product containing CSN5 promoter and GATA1 predicted binding site 1 (GGATGT).

According to the protocol of Experiment 8, each well of 96-well plate was added with $100 \mu \mathrm{L}$ reporter gene cell lysate, followed by centrifugation at $15000 \mathrm{rpm}$ for 5 min to collect the supernatant. The sample was detected by a microplate reader with an interval of $2 \mathrm{~s}$ and a measurement time of $10 \mathrm{~s}$. Then $100 \mu \mathrm{L}$ luciferase detection reagent was added into $100 \mu \mathrm{L}$ sample for RLU measurement.

\section{Chromatin immunoprecipitation assay (ChIP)}

The cells were treated with $1 \%$ paraformaldehyde and incubated at $37^{\circ} \mathrm{C}$ for $10 \mathrm{~min}$. The cells were resuspended in SDS lysis buffer and broken by ultrasonic, and the supernatant was collected. CHIP diluent was added with protease inhibitor, and this solution was used to dilute the cell supernatant. Then $200 \mu \mathrm{L}$ cell supernatant was added into $1800 \mu \mathrm{L}$ ChIP diluent, making the final volume as $2 \mathrm{~mL}$. The $10 \mu \mathrm{L}$ cell supernatant dilution was regard as input control. Protein A Agarose/salmon sperm DNA was added to the supernatant, followed by antibody (GATA1) incubation overnight. Then $200 \mu \mathrm{L}$ eluent was added to the protein A Agarose/antibody complex, and the supernatant was collected. Next, $20 \mu \mathrm{L} 5 \mathrm{MNaCl}$ was added to each $500 \mu \mathrm{L}$ eluent and subjected to water bath at $65^{\circ} \mathrm{C}$ for $4 \mathrm{~h}$, followed by $10 \mu \mathrm{L} 0.5 \mathrm{M}$ EDTA, $20 \mu \mathrm{L}$ $1 \mathrm{M}$ Tris- $\mathrm{HC} 1$ and $2 \mu \mathrm{L}$ proteinase $\mathrm{K}$ at a $45^{\circ} \mathrm{C}$-water bath for $1 \mathrm{~h}$. After filtered by DNA clean-up Column, the fluid was collected, and the qPCR was performed as described as 3.6.

\section{Construction of lentivirus}

Based on the construction of vector and identification of Colony PCR (Supplementary Figure 1), the bacterial solution with correct sequencing was seeded into $10 \mathrm{~mL}$ LB liquid medium containing Amp antibiotic and incubated, and then the plasmid was extracted. The GT1-7 cells were seeded into a 24-well plate and prepared for transfection. Then $0.8 \mu \mathrm{g}$ plasmid was added to $50 \mu \mathrm{L}$ Opti-MEM medium (GIBCO, Grand Island, USA) or $2.0 \mu \mathrm{L}$ lipofectamine 2000 (Thermo Fisher Scientific, NY, USA) at room temperature for $5 \mathrm{~min}$, respectively. The two diluents were mixed for 5 min, and then $100 \mu \mathrm{L}$ mixture was added into each well of the cell plate for $12 \mathrm{~h}$-incubation at $37^{\circ} \mathrm{C}$. After $72 \mathrm{~h}$ of transfection, the infective efficiency could be determined.

\section{Statistical analysis}

All data were expressed as means \pm SEM. Assessment of statistical significance was done using statistical analysis software SPSS (IBM SPSS Statistics 21; SPSS Inc., Chicago, IL, USA). For comparisons between experimental groups, one-way ANOVA and Tukey's test were applied. All the $\mathrm{P}$ values were two-sided and the differences were considered statistically significant at $\mathrm{P}<0.05$.

\section{Ethical statement}

The experimental operations were approved by the Animal Ethics Committee of Nanjing Chinese Medicine University (approval No: SYXK (Su) 2014-0001). 


\section{AUTHOR CONTRIBUTIONS}

Huifang Zhou finished study design, Hua Feng, Jianxia Lu, Qing Zhang finished experimental studies, Hua Feng, Xingran Tang Yujie Shang finished data analysis, Hua Feng finished manuscript editing. All authors read and approved the final manuscript.

\section{ACKNOWLEDGMENT}

We would like to acknowledge the reviewers for their helpful comments on this paper.

\section{CONFLICTS OF INTEREST}

The authors declare that they have no conflicts of interest.

\section{FUNDING}

Sponsored by Project of National Natural Science Foundation of China (No. 81473713 and 81774354); Phase III Project of Advantageous Discipline of Traditional Chinese Medicine (No. ZYX03KF005); Leading Talents of Traditional Chinese Medicine in Jiangsu Province (No. SLJ0202).

\section{Editorial note}

${ }^{\&}$ This corresponding author has a verified history of publications using a personal email address for correspondence.

\section{REFERENCES}

1. Bernard V, Young J, Binart N. Prolactin - a pleiotropic factor in health and disease. Nat Rev Endocrinol. 2019; 15:356-65.

https://doi.org/10.1038/s41574-019-0194-6 PMID: 30899100

2. Arefi S, Fallah N. Comparison of pct after vaginal and oral bromocriptine use in women with hyperprolactinemia and infertility. J Reprod Infertil. 2002; 3:36-41.

3. Capozzi A, Scambia G, Pontecorvi A, Lello S. Hyperprolactinemia: pathophysiology and therapeutic approach. Gynecol Endocrinol. 2015; 31:506-10. https://doi.org/10.3109/09513590.2015.1017810 PMID:26291795

4. Gasco V, Grottoli S. Physiopathology, Diagnosis, and Treatment of Hyperprolactinemia. In: Casanueva F, Ghigo E, editors. Hypothalamic-Pituitary Diseases. Endocrinology. Cham: Springer. 2018. https://doi.org/10.1007/978-3-319-44444-4_15
5. Li L, Jiang H, Wei X, Geng D, He M, Du H. Bu Shen Zhu Yun Decoction Improves Endometrial Receptivity via VEGFR-2-Mediated Angiogenesis. Evid Based Complement Alternat Med. 2019; 2019:3949824. https://doi.org/10.1155/2019/3949824 PMID:32082394

6. Bei L, Hui-fang ZH, Bo-ru ZH, Xiao-fei JI, Jian-ya XU, Tong XI, Jin-jun SH. Regulation of BuShen ZhuYun Decotion on gonadotropin in rat pituitary cells. Journal of Medical Postgraduates. 2018; 703-08.

7. Jiang X, Zhou H, Shi M, Zhou B, Liu B, Yuan Y, Shan J, $\mathrm{Xu} J$, Xie T. Bu-shen-zhu-yun decoction promotes synthesis and secretion of FSH $\beta$ and $\mathrm{LH} \beta$ in anterior pituitary cells in vitro. Biomed Pharmacother. 2018; 102:494-501.

https://doi.org/10.1016/i.biopha.2018.02.003 PMID:29579710

8. Harter CJ, Kavanagh GS, Smith JT. The role of kisspeptin neurons in reproduction and metabolism. J Endocrinol. 2018; 238:R173-83. https://doi.org/10.1530/JOE-18-0108 PMID:30042117

9. Trevisan $\mathrm{CM}$, Montagna $\mathrm{E}$, de Oliveira R, Christofolini DM, Barbosa CP, Crandall KA, Bianco B. Kisspeptin/GPR54 System: What Do We Know About Its Role in Human Reproduction? Cell Physiol Biochem. 2018; 49:1259-76.

https://doi.org/10.1159/000493406

PMID:30205368

10. Sinha S, Sharma S, Vora J, Shah H, Srivastava A, Shrivastava N. Mucuna pruriens (L.) DC chemo sensitize human breast cancer cells via downregulation of prolactin-mediated JAK2/STAT5A signaling. J Ethnopharmacol. 2018; 217:23-35.

https://doi.org/10.1016/j.jep.2018.02.006 PMID:29427634

11. Ried K, Stuart K. Efficacy of Traditional Chinese Herbal Medicine in the management of female infertility: a systematic review. Complement Ther Med. 2011; 19:319-31.

https://doi.org/10.1016/j.ctim.2011.09.003

PMID:22036524

12. Wen-Jie $Y$, Jing $Y$, Tai-Lang $Y$. [Effects of zhuyun recipe on the endometrial receptivity in mice with blastocyst implantation dysfunction and ovulation stimulation]. Zhongguo Zhong Xi Yi Jie He Za Zhi. 2012; 32:1554-57. PMID:23359985

13. Yu N, Yang J, Yin T. Extracts from a traditional Chinese herbal remedy (Zhuyun recipe) improve endometrial receptivity in mice with embryonic implantation dysfunction and ovulation stimulation. J Ethnopharmacol. 2011; 137:389-95. 
https://doi.org/10.1016/j.jep.2011.05.037

PMID:21740961

14. Zhou HF, Li AP, Tan Y. [Effects of bushen zhuyun recipe on protein expressions of estrogen receptor, progesterone receptor and integrin alpha5 and beta3 in endometrium of rats at the implantation stage]. Zhongguo Zhong Xi Yi Jie He Za Zhi. 2009; 29:628-31.

PMID:19852297

15. Borba VV, Zandman-Goddard G, Shoenfeld Y. Prolactin and Autoimmunity. Front Immunol. 2018; 9:73.

https://doi.org/10.3389/fimmu.2018.00073

PMID:29483903

16. Kataria J, Gill GK, Kaur M. Interrelationship of thyroid hormones, obesity, and prolactin in infertile women. Asian J Pharm Clin Res. 2018; 11:136. https://doi.org/10.22159/ajpcr.2017.v11i1.20861

17. Salvatori R. Dopamine agonist withdrawal in hyperprolactinemia: when and how. Endocrine. 2018; 59:4-6.

https://doi.org/10.1007/s12020-017-1469-0

PMID:29124662
18. Goffin V, Touraine P. The prolactin receptor as a therapeutic target in human diseases: browsing new potential indications. Expert Opin Ther Targets. 2015; 19:1229-44.

https://doi.org/10.1517/14728222.2015.1053209

PMID:26063597

19. Clarke H, Dhillo WS, Jayasena CN. Comprehensive Review on Kisspeptin and Its Role in Reproductive Disorders. Endocrinol Metab (Seoul). 2015; 30:124-41. https://doi.org/10.3803/EnM.2015.30.2.124 PMID:26194072

20. Hu KL, Zhao H, Yu Y, Li R. Kisspeptin as a potential biomarker throughout pregnancy. Eur J Obstet Gynecol Reprod Biol. 2019; 240:261-66.

https://doi.org/10.1016/j.ejogrb.2019.07.016

PMID:31344665 


\section{SUPPLEMENTARY MATERIALS}

\section{Supplementary Figures}

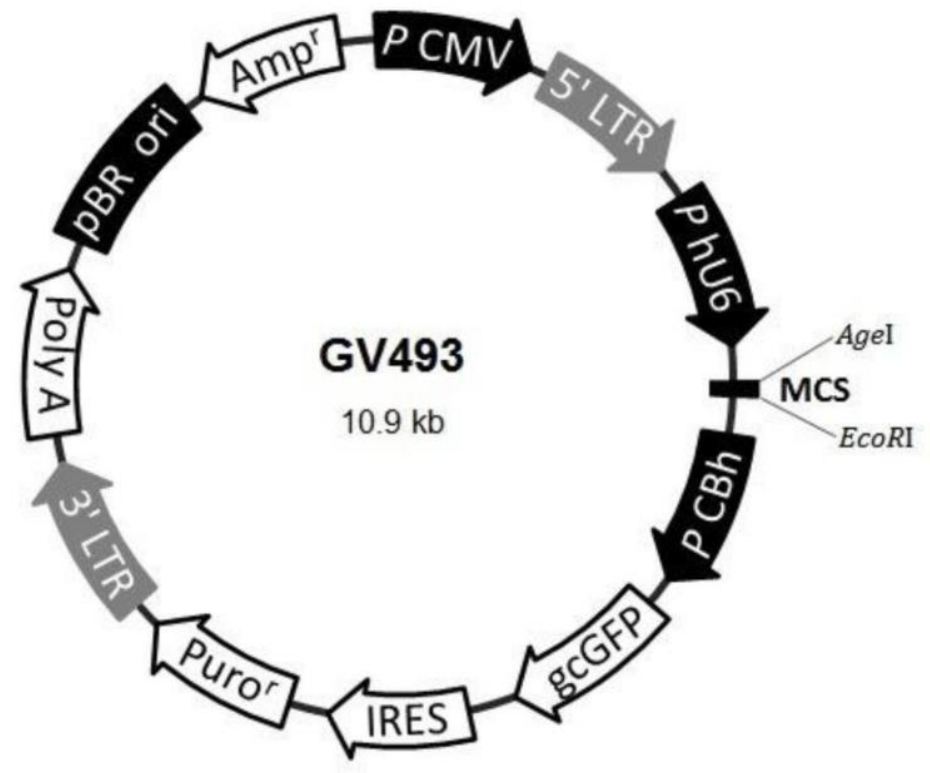

Supplementary Figure 1. The schematic diagram of lentiviral vector.

A

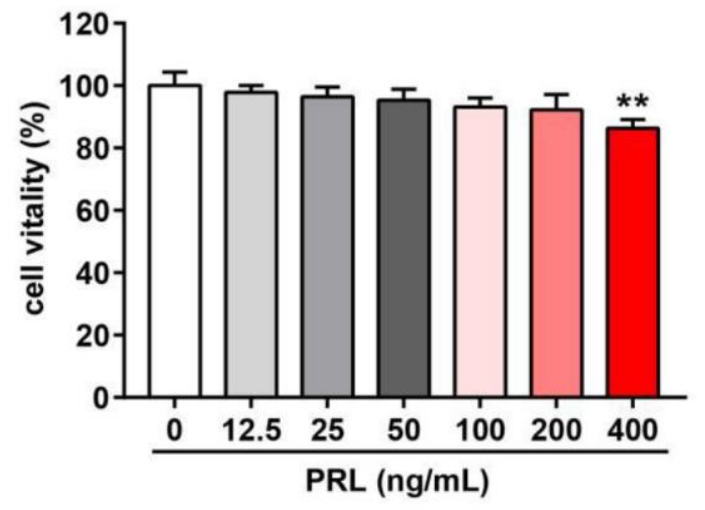

B

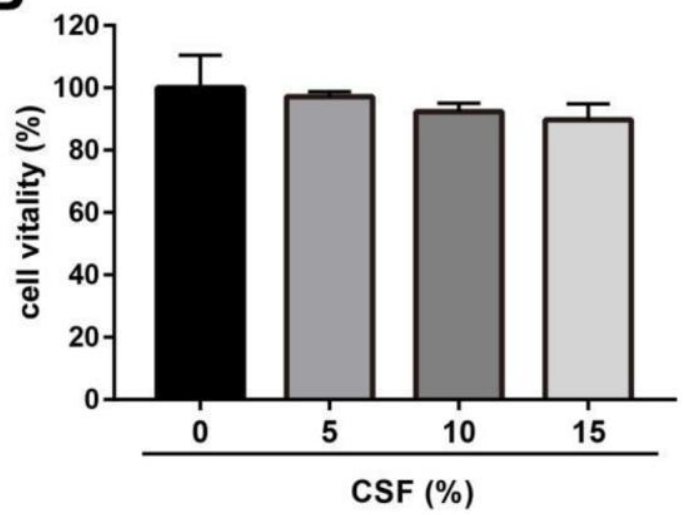

Supplementary Figure 2. Inhibitory effect of PRL or CSF in GT1-7 cells. (A) The change of cell vitality effected by different PRL concentration levels. (B) The change of cell vitality effected by different CSF concentration levels. Results were expressed as mean \pm SD ( $n=3$ ). $* * p<0.01$ vs Control group. 
A

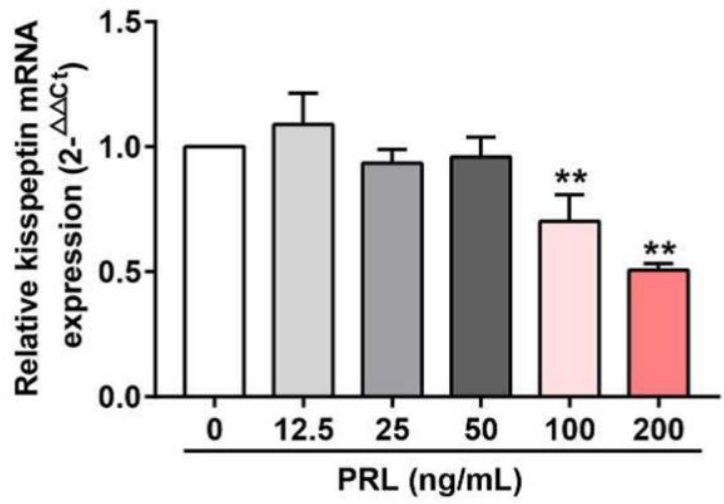

B

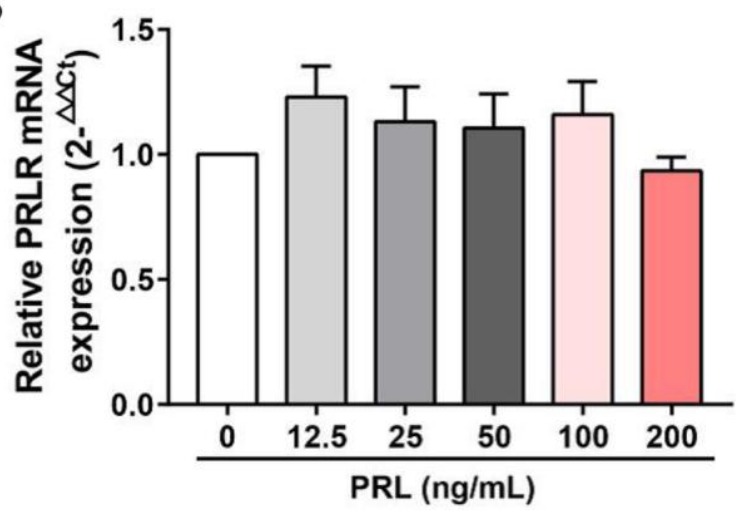

Supplementary Figure 3. Effect of the mRNA expressions of PRLR and kisspeptin in GT1-7. The mRNA expressions of PRLR and kisspeptin were detected by RT-PCR. The mRNA expressions of PRLR (A) and kisspeptin (B) were normalized to control. The results were presented as mean $\pm S D(n=3)$. ${ }^{* *} p<0.01$, vs. Control group.
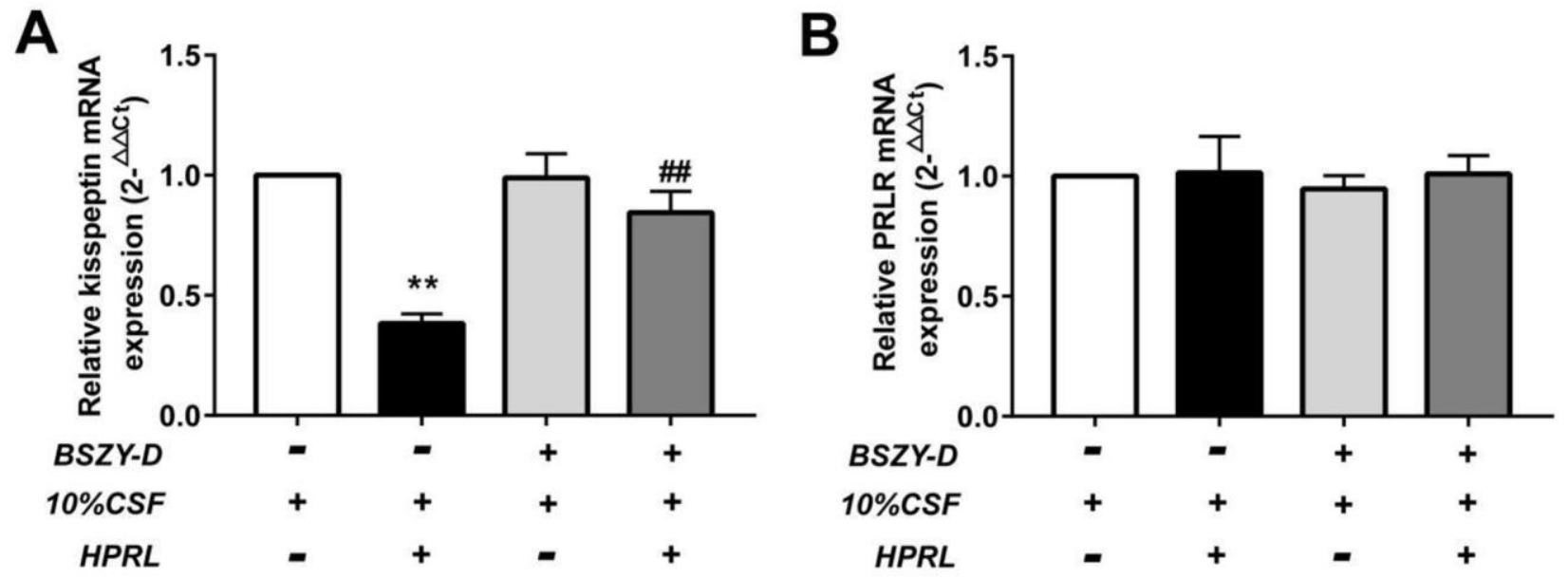

Supplementary Figure 4. Effect of the mRNA expressions of PRLR and kisspeptin in GT1-7. The mRNA expressions of PRLR and kisspeptin were detected by RT-PCR. The mRNA expressions of PRLR (A) and kisspeptin (B) were normalized to control. The results were presented as mean $\pm S D(n=3) .{ }^{*} p<0.01$ vs. Control group, \#\#p $<0.01$ vs. HPRL group. 


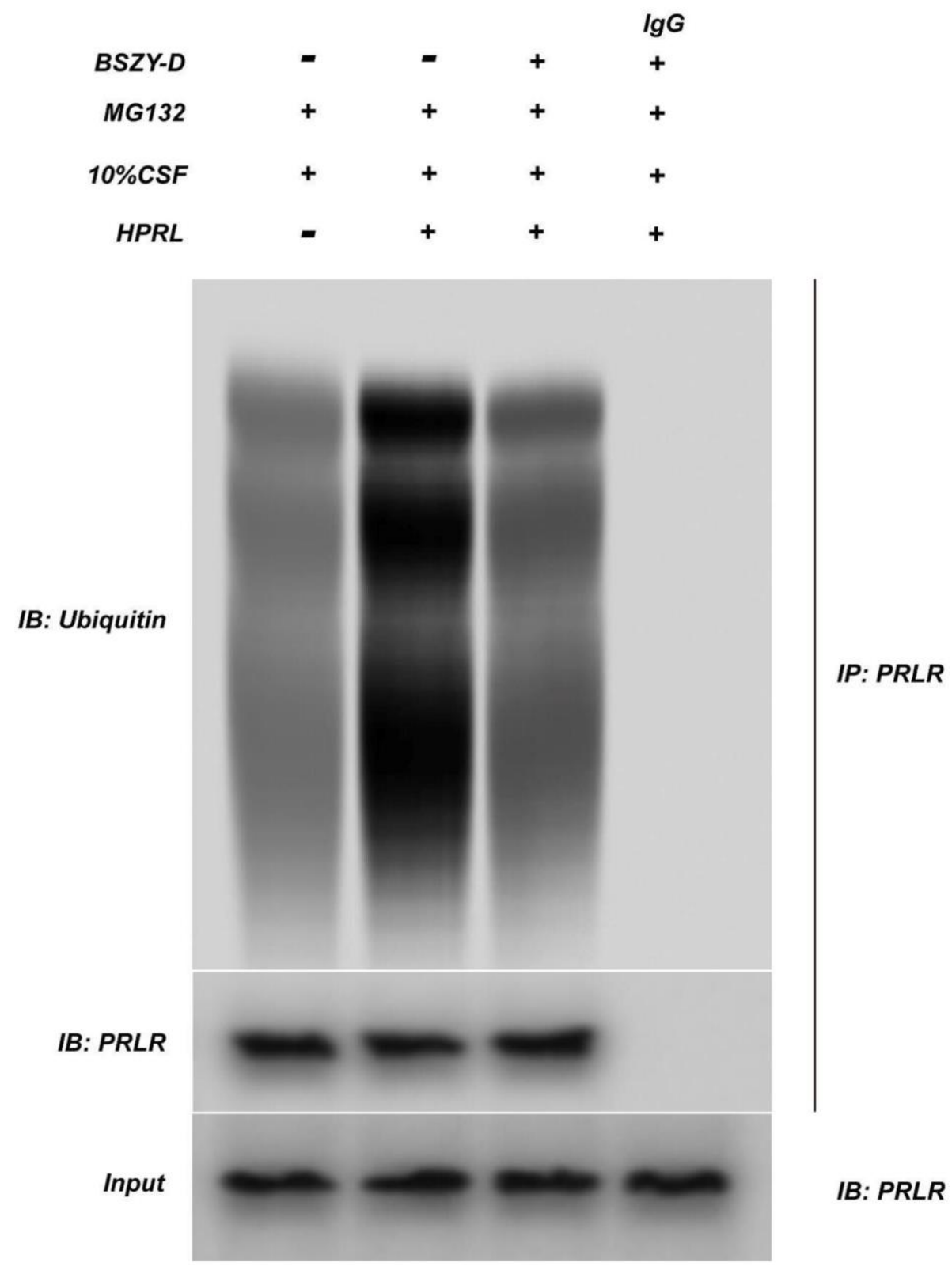

Supplementary Figure 5. The ubiquitin level of PRLR was measured with Co-IP. $(n=3)$. 


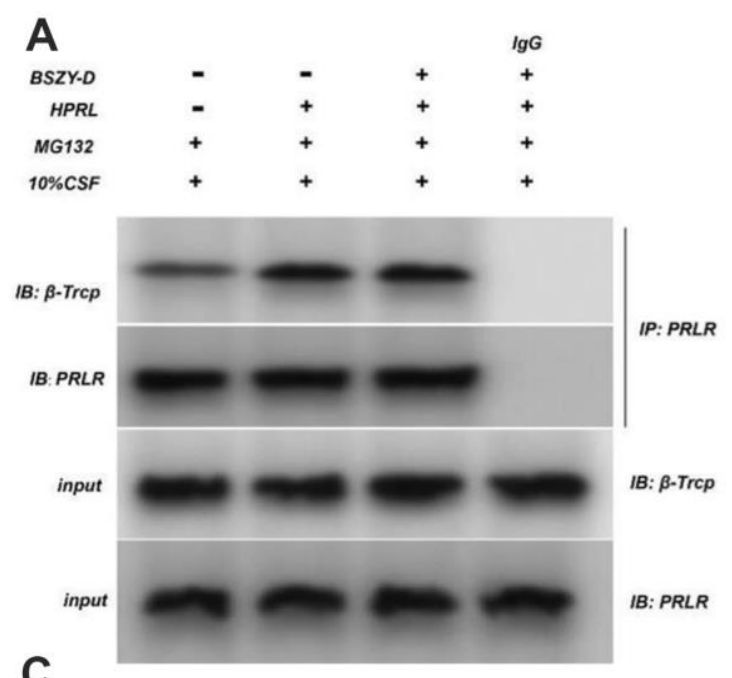

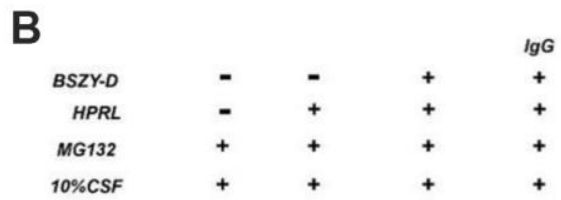
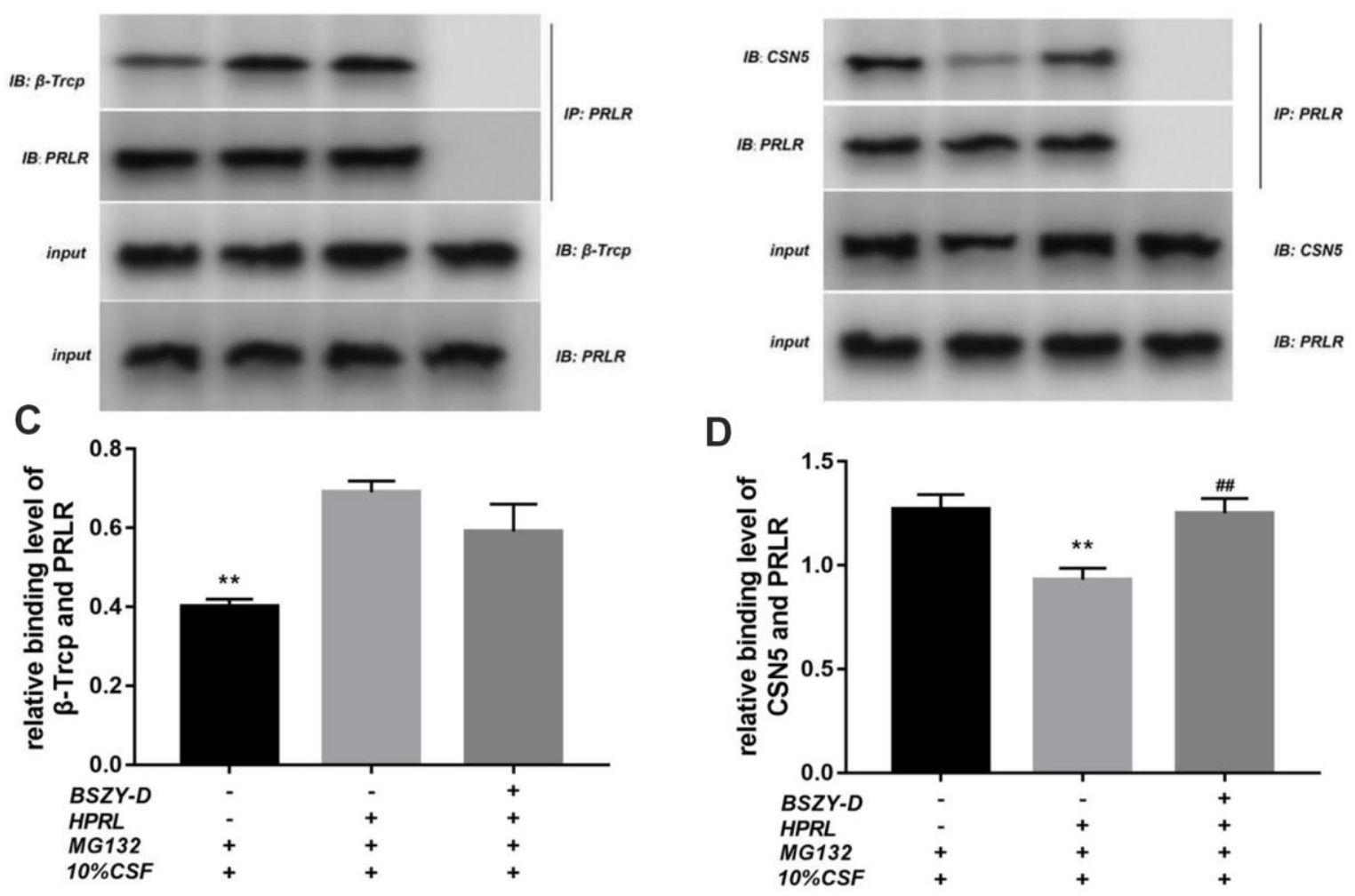

Supplementary Figure 6. The level of $\boldsymbol{\beta}$-Trcp, PRLR, and CSN5 were measured with Co-IP ( $\mathbf{n}=\mathbf{3}$ ). (A) Representative blots of Co-IP between PRLR and $\beta$-Trcp. (B) Representative blots of Co-IP between PRLR and CSN5. (C) Quantitative relative binding level of RLR and $\beta$-Trcp. (D) Quantitative relative binding level of RLR and CSN5.

A

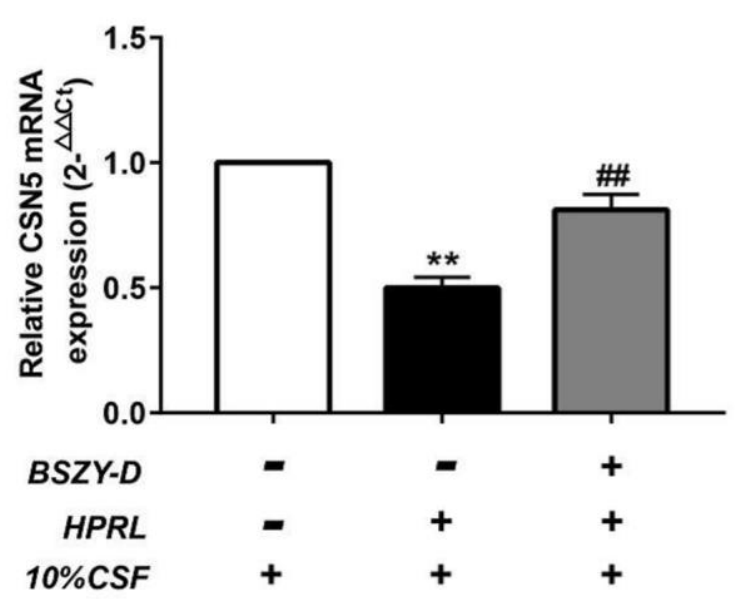

B

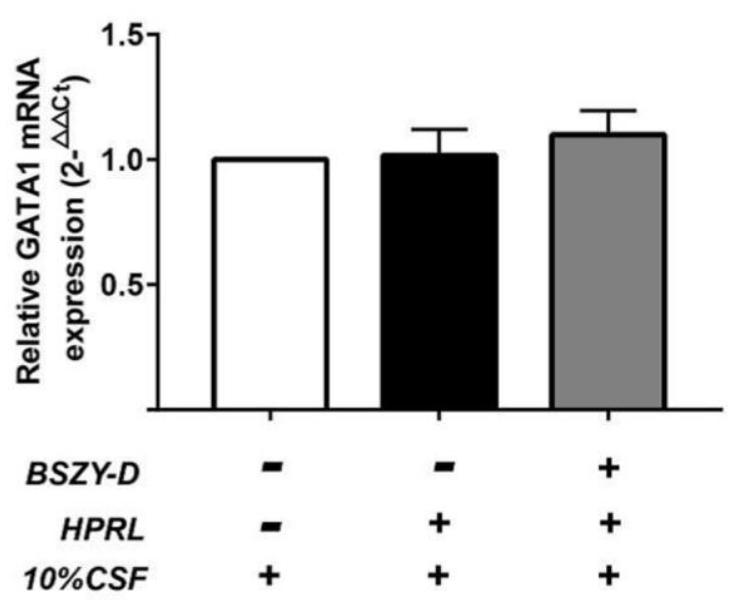

Supplementary Figure 7. Effect of the mRNA expressions of CSN5and GATA1 in GT1-7. The mRNA expressions of CSN5 and GATA1 were detected by RT-PCR. The mRNA expressions of CSN5 (A) and GATA1 (B) were normalized to control. The results were presented as mean $\pm S D(n=3) .{ }^{* *} p<0.01$ vs. Control group, \#\#p $<0.01$ vs. HPRL group. 

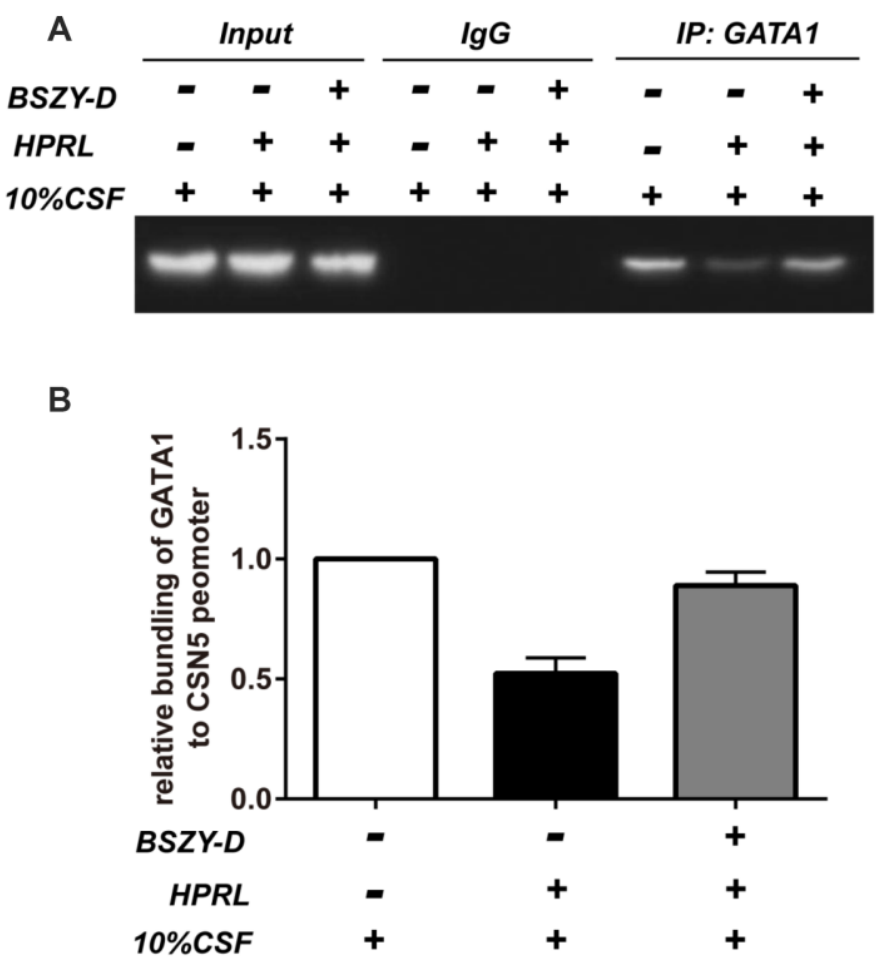

Supplementary Figure 8. Chromatin immunoprecipitation (ChIP) assay on the promoter of GATA1. (A) Representative blot of ChIP assay on GATA1. (B) Quantitative relative binding of GATA1 to CSN5 promoter. The results were presented as mean \pm SD $(n=3) .{ }^{* *} p<$ 0.01 vs. Control group, \#\#p < 0.01 vs. HPRL group.
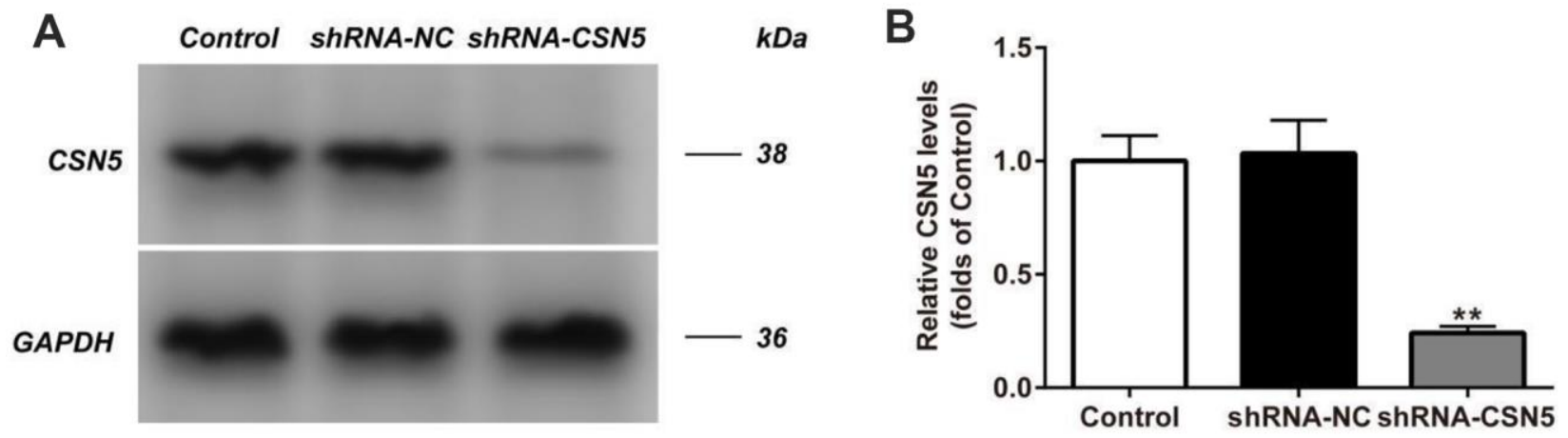

Supplementary Figure 9. The level of CSN5 in GT1-7 was detected by western blot assay and representative bands were shown in (A). The level of CSN5 (B) was normalized to control. The results were presented as mean $\pm \operatorname{SD}(n=3)$. ${ }^{* *} p<0.01$ vs. shRNA-NC group. 


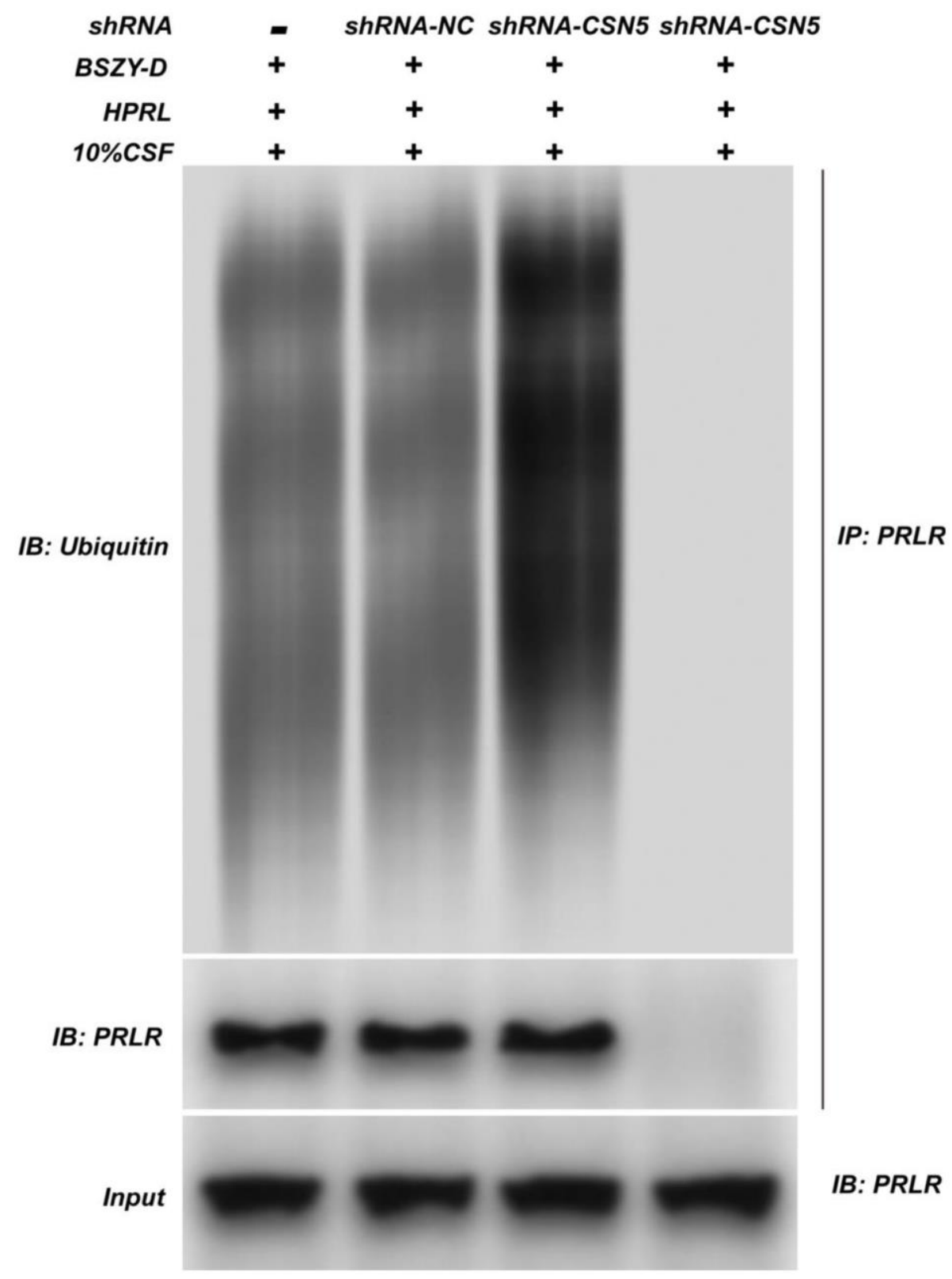

Supplementary Figure 10. The ubiquitin level of PRLR was measured with Co-IP ( $n=3)$. 